\title{
APPROXIMATIONS AND THE LINEARITY OF THE SHEPP SPACE
}

\author{
Aoi HONDA, Yoshiaki OKAZAKI and Hiroshi SATO \\ (Received 10 April 2014) \\ In memory of Professor L. A. Shepp
}

\begin{abstract}
Suggested by Shepp [Ann. Math. Statist. 36(4) (1965), 1107-1112] we defined a sequence space $\Lambda_{p}(f)$ determined by a single function $f(\neq 0) \in L_{p}(\mathbb{R}, d x), 1 \leq p<+\infty$, and discussed the structure of it. The problems are the linearity and the visible sequential representation of $\Lambda_{p}(f)$. In this paper we name $\Lambda_{p}(f)$ a Shepp space and discuss the problems in the case of $p=2$ by defining an inner approximation $\Lambda_{2}^{0}(f)$ and an outer approximation $\Lambda_{2}^{\varphi}(f)$ of $\Lambda_{2}(f)$, and we give a necessary and sufficient condition for $\Lambda_{2}^{0}(f)=\Lambda_{2}^{\varphi}(f)$ in terms of doubling dimension. In this case $\Lambda_{2}(f)$ is a linear space and those approximations are its visible sequential representations. We also give an example such that $\Lambda_{2}(f)$ is a linear space but $\Lambda_{2}^{0}(f) \neq \Lambda_{2}^{\varphi}(f)$.
\end{abstract}

\section{Introduction}

A Shepp space for $f(\neq 0) \in L_{p}(\mathbb{R}, d x), 1 \leq p<+\infty$, is a sequence space

$$
\Lambda_{p}(f):=\left\{\boldsymbol{a}:=\left\{a_{k}\right\} \in \mathbb{R}^{\infty}\left|\sum_{k=1}^{+\infty} \int_{-\infty}^{+\infty}\right| f\left(x-a_{k}\right)-\left.f(x)\right|^{p} d x<+\infty\right\},
$$

which is a complete separable additive group with a translation invariant metric

$$
d_{p}^{f}(\boldsymbol{a}, \boldsymbol{b}):=\left\{\sum_{k=1}^{+\infty} \int_{-\infty}^{+\infty}\left|f\left(x-a_{k}\right)-f\left(x-b_{k}\right)\right|^{p} d x\right\}^{1 / p}, \quad \boldsymbol{a}, \boldsymbol{b} \in \mathbb{R}^{\infty},
$$

and included in $\ell_{p}$ (see [2]).

Such a space first appeared in Shepp [7] as $\Lambda_{2}(\sqrt{\rho})$ where $\rho$ is a probability density, and had been studied by many authors concerned with the absolute continuity of the translation of infinite product measures $\left[\mathbf{5 , 8 , 9}\right.$. Chatterji and Mandrekar [1] gave an example of $\Lambda_{2}(\sqrt{\rho})$ which is not a linear space.

Suggested by the Shepp's paper, we defined and studied the sequence space $\Lambda_{p}(f)$ (see [2-4]), and we name it a Shepp space in this paper. Nakamura and Hashimoto gave other examples of nonlinear Shepp spaces [6].

Before stating the results of the paper, we prepare several notations and terminologies.

2010 Mathematics Subject Classification: Primary 46A45; Secondary 46A16, 46B20.

Keywords: Shepp space; linearity; doubling condition; doubling dimension; sequential representation.

(C) 2015 Faculty of Mathematics, Kyushu University 
A non-negative function $\varphi$ on $[0,+\infty)$ is said to satisfy the doubling condition if there exists $h \in \mathbb{R}$ such that

$$
D(h ; \varphi):=\limsup _{\substack{x \rightarrow+\infty \\ \varphi(x)>0}} \sup _{T \geq 1} \frac{\varphi(T x)}{\varphi(x) T^{h}}<+\infty,
$$

and

$$
H(\varphi):=\inf \{h \in \mathbb{R} \mid D(h ; \varphi)<+\infty\}
$$

is called the doubling dimension of $\varphi$, where $\inf \emptyset:=+\infty$ (see [3]).

Let $\mathcal{L}$ be a set. Then a function $\gamma: \mathcal{L} \times \mathcal{L} \rightarrow[0,+\infty)$ is called a quasi-metric on $\mathcal{L}$ if $\gamma$ satisfies:

(QM1) $\gamma(\boldsymbol{a}, \boldsymbol{b})=0$ if and only if $\boldsymbol{a}=\boldsymbol{b}, \quad \boldsymbol{a}, \boldsymbol{b} \in \mathcal{L}$;

$(\mathrm{QM} 2) \gamma(\boldsymbol{a}, \boldsymbol{b})=\gamma(\boldsymbol{b}, \boldsymbol{a}), \quad \boldsymbol{a}, \boldsymbol{b} \in \mathcal{L} ;$ and

(QM3) there exists $A \geq 1$ such that

$$
\gamma(\boldsymbol{a}, \boldsymbol{c}) \leq A[\gamma(\boldsymbol{a}, \boldsymbol{b})+\gamma(\boldsymbol{b}, \boldsymbol{c})], \quad \boldsymbol{a}, \boldsymbol{b}, \boldsymbol{c} \in \mathcal{L} .
$$

The quasi-metrics $\gamma$ and $\gamma^{\prime}$ on a set $\mathcal{L}$ are said to be equivalent if there exists $B \geq 1$ such that

$$
\frac{1}{B} \gamma^{\prime}(\boldsymbol{a}, \boldsymbol{b}) \leq \gamma(\boldsymbol{a}, \boldsymbol{b}) \leq B \gamma^{\prime}(\boldsymbol{a}, \boldsymbol{b}), \quad \boldsymbol{a}, \boldsymbol{b} \in \mathcal{L} .
$$

Two equivalent quasi-metrics $\gamma$ and $\gamma^{\prime}$ give the same topology on $\mathcal{L}$.

Let $\mathcal{L}$ be a linear space. Then a quasi-metric $\gamma$ on $\mathcal{L}$ is said to be a linear quasi-metric if $(\mathcal{L}, \gamma)$ is a topological linear space, and translation invariant if $\gamma$ satisfies:

(TRL) $\gamma(\boldsymbol{a}, \boldsymbol{b})=\gamma(\boldsymbol{a}-\boldsymbol{b}, \mathbf{0}), \quad \boldsymbol{a}, \boldsymbol{b} \in \mathcal{L}$.

For $f(\neq 0) \in L_{2}$ we define a function

$$
\varphi_{f}(x):=\int_{0}^{x} \alpha^{2}|\hat{f}(\alpha)|^{2} d \alpha, \quad x \geq 0,
$$

where $\hat{f}(\alpha):=(1 / \sqrt{2 \pi}) \int e^{-i \alpha x} f(x) d x$ is the Fourier transform of $f$.

Although the definitions of $\Lambda_{p}(f)$ and $d_{p}^{f}$ do not have any ambiguities, the visible representations of them in terms of sequences are not clear. We call such a representation $a$ visible sequential representation. Problems are to give the visible sequential representations of $\left(\Lambda_{p}(f), d_{p}^{f}\right)$, and to characterize the linearity of Shepp spaces $\Lambda_{p}(f)$. We proved that if $f$ is a piecewise monotone function, then $\Lambda_{p}(f)$ is a linear space [3, Theorem 2.1]. In [4] we discussed the inclusion relations among the Shepp spaces and proved that they are always continuous.

In this paper we discuss the visible sequential representations and the linearity of the Shepp spaces in the case of $p=2$, by introducing inner and outer approximations of $\left(\Lambda_{2}(f), d_{2}^{f}\right)$.

In Section 2, for $f(\neq 0) \in L_{2}$, the inner approximation space $\Lambda_{2}^{0}(f) \subset \Lambda_{2}(f)$ is defined by

$$
\Lambda_{2}^{0}(f):=\left\{\boldsymbol{a}=\left\{a_{k}\right\} \in \mathbb{R}^{\infty} \mid \sum_{k=1}^{+\infty} \int_{1 /\left|a_{k}\right|}^{+\infty} \frac{1}{\alpha^{3}} \varphi_{f}(\alpha) d \alpha<+\infty\right\},
$$


and

$$
\gamma_{f}(\boldsymbol{a}, \boldsymbol{b}):=\sqrt{\sum_{k=1}^{+\infty} \int_{1 /\left|a_{k}-b_{k}\right|}^{+\infty} \frac{1}{\alpha^{3}} \varphi_{f}(\alpha) d \alpha}, \quad \boldsymbol{a}=\left\{a_{k}\right\}, \quad \boldsymbol{b}=\left\{b_{k}\right\} \in \mathbb{R}^{\infty}
$$

defines a translation invariant linear quasi-metric on $\Lambda_{2}^{0}(f)$ (Theorem 6). Here $\Lambda_{2}^{0}(f)$ is the maximum linear space in $\Lambda_{2}(f)$, and if $\Lambda_{2}(f)$ is a linear space then we have $\Lambda_{2}(f)=\Lambda_{2}^{0}(f)$ and $\gamma_{f}$ is equivalent to $d_{2}^{f}$ (Theorem 10), that means $\left(\Lambda_{2}^{0}(f), \gamma_{f}\right)$ is a visible sequential representation of $\left(\Lambda_{2}(f), d_{2}^{f}\right)$.

In Section 3, for $f(\neq 0) \in L_{2}$, the outer approximation space $\Lambda_{2}^{\varphi}(f) \supset \Lambda_{2}(f)$ is defined by

$$
\Lambda_{2}^{\varphi}(f):=\left\{\boldsymbol{a}=\left\{a_{k}\right\} \in \mathbb{R}^{\infty} \mid \sum_{k=1}^{+\infty} a_{k}^{2}\left[1+\varphi_{f}\left(\frac{1}{\left|a_{k}\right|}\right)\right]<+\infty\right\}
$$

(see [3]). A function $\delta_{f}$ given by

$$
\begin{gathered}
\delta_{f}(\boldsymbol{a}, \boldsymbol{b}):=\sqrt{\sum_{k=1}^{+\infty}\left[\left|a_{k}-b_{k}\right| \wedge 1\right]^{2}\left[1+\varphi_{f}\left(\frac{1}{\left|a_{k}-b_{k}\right| \wedge 1}\right)\right],} \\
\boldsymbol{a}=\left\{a_{k}\right\}, \boldsymbol{b}=\left\{b_{k}\right\} \in \mathbb{R}^{\infty},
\end{gathered}
$$

where $x \wedge y:=\min \{x, y\}$, defines a topology on $\Lambda_{2}^{\varphi}(f)$ and the inclusion $\Lambda_{2}(f) \subset \Lambda_{2}^{\varphi}(f)$ is continuous [3]. In general, $\Lambda_{2}^{\varphi}(f)$ is not a linear space nor is $\delta_{f}$ a quasi-metric. We prove that $\Lambda_{2}^{\varphi}(f)$ is a linear space if and only if $D\left(2 ; \varphi_{f}\right)<+\infty$ (Theorem 16 and Lemma 18). In this case, $\delta_{f}$ is a linear quasi-metric on $\Lambda_{2}^{\varphi}(f)$ (Theorem 20).

In Section 4, we discuss the condition for $\Lambda_{2}^{0}(f)=\Lambda_{2}^{\varphi}(f)$, which implies the linearity of $\Lambda_{2}(f)$. In [3], we showed that $H\left(\varphi_{f}\right)<2$ implies $\Lambda_{2}^{0}(f)=\Lambda_{2}^{\varphi}(f)$. In this paper we prove the converse, that is, $\Lambda_{2}^{0}(f)=\Lambda_{2}^{\varphi}(f)$ implies $H\left(\varphi_{f}\right)<2$ (Theorem 24). In this case, $\delta_{f}$ is a linear quasi-metric equivalent to $d_{2}^{f}$ (Theorem 23) and $\left(\Lambda_{2}^{\varphi}(f), \delta_{f}\right)$ is a visible sequential representation of $\left(\Lambda_{2}(f), d_{2}^{f}\right)$.

In Section 5, we remark that $\Lambda_{2}^{0}(f)=\Lambda_{2}^{\varphi}(f)$ is not a necessary condition for the linearity of $\Lambda_{2}(f)$. We give an illuminating example such that both $\Lambda_{2}(f)$ and $\Lambda_{2}^{\varphi}(f)$ are linear spaces but $\Lambda_{2}^{0}(f) \neq \Lambda_{2}^{\varphi}(f)$.

Through the discussion Lemma 4 plays crucial roles.

\section{Inner approximation $\left(\Lambda_{2}^{0}(f), \gamma_{f}\right)$}

In this section, we discuss the inner approximation $\left(\Lambda_{2}^{0}(f), \gamma_{f}\right)$.

Lemma 1. For $f(\neq 0) \in L_{2}$, define

$$
\xi_{f}(t):= \begin{cases}\int_{1 /|t|}^{+\infty} \frac{1}{\alpha^{3}} \varphi_{f}(\alpha) d \alpha, & t \neq 0 \\ 0, & t=0 .\end{cases}
$$

Then we have

$$
\xi_{f}(t)=\frac{1}{2}\left\{t^{2} \varphi_{f}\left(\frac{1}{|t|}\right)+\int_{1 /|t|}^{+\infty}|\hat{f}(\alpha)|^{2} d \alpha\right\},
$$


and $\left(1 / \alpha^{3}\right) \varphi_{f}(\alpha)$ is integrable. Furthermore, for any fixed $\boldsymbol{a}=\left\{a_{k}\right\} \in \Lambda_{2}^{0}(f)$, the function

$$
\gamma_{f}(t \boldsymbol{a}, \mathbf{0})^{2}=\sum_{k} \xi_{f}\left(t a_{k}\right)
$$

is continuous in $t \in[-1,1]$.

Proof. We remark that $\lim _{R \rightarrow+\infty}\left(1 / R^{2}\right) \varphi_{f}(R)=0$ (see [3, Lemma 4.4]) and $\lim _{\varepsilon \rightarrow 0+}$ $\left(1 / \varepsilon^{2}\right) \varphi_{f}(\varepsilon) \leq \lim _{\varepsilon \rightarrow 0+} \int_{0}^{\varepsilon}|\hat{f}(\alpha)|^{2} d \alpha=0$, which imply ( $\left.\boldsymbol{\Xi}\right)$ by integration by parts. For $\boldsymbol{a}=\left\{a_{k}\right\} \in \Lambda_{2}^{0}(f)$ we have

$$
\gamma_{f}(t \boldsymbol{a}, \mathbf{0})^{2}=\sum_{k} \xi_{f}\left(t a_{k}\right) \leq \sum_{k} \xi_{f}\left(a_{k}\right)=\gamma_{f}(\boldsymbol{a}, \mathbf{0})^{2}<+\infty, \quad t \in[-1,1]
$$

and by the dominated convergence theorem, $\gamma_{f}(t \boldsymbol{a}, \mathbf{0})$ is continuous in $t \in[-1,1]$.

LEMMA 2. For $f(\neq 0) \in L_{2}, \Lambda_{2}^{0}(f)$ is the maximum linear space in $\Lambda_{2}(f)$. Therefore, we have $\Lambda_{2}(f)=\Lambda_{2}^{0}(f)$ if and only if $\Lambda_{2}(f)$ is a linear space.

Proof. By [3, Lemma 2.4], for $\boldsymbol{a}=\left\{a_{k}\right\} \in \mathbb{R}^{\infty}$ we have $t \boldsymbol{a} \in \Lambda_{2}(f)$ for any $t \in \mathbb{R}$ if and only if $\boldsymbol{a}$ satisfies

$$
\sum_{k} a_{k}^{2} \varphi\left(\frac{1}{\left|a_{k}\right|}\right)<+\infty \text { and } \sum_{k} \int_{1 /\left|a_{k}\right|}^{+\infty}|\hat{f}(\alpha)|^{2} d \alpha<+\infty .
$$

On the other hand, by ( $\boldsymbol{\Xi})$, for $\boldsymbol{a} \in \mathbb{R}^{\infty}, \boldsymbol{a} \in \Lambda_{2}^{0}(f)$ if and only if $\boldsymbol{a}$ satisfies (S). Consequently for $\boldsymbol{a} \in \mathbb{R}^{\infty}, \boldsymbol{a} \in \Lambda_{2}^{0}(f)$ if and only if $t \boldsymbol{a} \in \Lambda_{2}(f)$ for any $t \in \mathbb{R}$. In particular, if $\boldsymbol{a} \in \Lambda_{2}^{0}(f)$, then we have $\boldsymbol{a} \in \Lambda_{2}(f)$ which shows $\Lambda_{2}^{0}(f) \subset \Lambda_{2}(f)$.

For any $\boldsymbol{a} \in \Lambda_{2}^{0}(f)$ we have $(s t) \boldsymbol{a}=s(t \boldsymbol{a}) \in \Lambda_{2}(f)$ for any $s, t \in \mathbb{R}$, which implies $t \boldsymbol{a} \in \Lambda_{2}^{0}(f)$ and $\Lambda_{2}^{0}(f)$ is closed under the scalar multiplication.

We show that $\Lambda_{2}^{0}(f)$ is an additive group. Assume that $\boldsymbol{a}, \boldsymbol{b} \in \Lambda_{2}^{0}(f)$. Then we have $t \boldsymbol{a}, t \boldsymbol{b} \in \Lambda_{2}(f)$ for any $t \in \mathbb{R}$. Since $\Lambda_{2}(f)$ is an additive group, we have $t(\boldsymbol{a}-\boldsymbol{b}) \in \Lambda_{2}(f)$ for any $t \in \mathbb{R}$, which implies $\boldsymbol{a}-\boldsymbol{b} \in \Lambda_{2}^{0}(f)$.

From the above arguments $\Lambda_{2}^{0}(f)$ is a linear space in $\Lambda_{2}(f)$.

Let $\mathcal{L}$ be a linear space in $\Lambda_{2}(f)$. Then $\boldsymbol{a} \in \mathcal{L}$ implies $t \boldsymbol{a} \in \mathcal{L} \subset \Lambda_{2}(f)$ for any $t \in \mathbb{R}$, which implies $\mathcal{L} \subset \Lambda_{2}^{0}(f)$.

PRoposition 3. For $f(\neq 0) \in L_{2}, \gamma_{f}(\boldsymbol{a}, \boldsymbol{b})$ is a translation invariant quasi-metric on $\Lambda_{2}^{0}(f)$.

The following lemma is crucial in proving the above proposition.

LEMMA 4. For non-negative functions $\Phi(x)$ and $\Psi(x)$ on a set $\mathfrak{X}$, assume that:

(1) $\Psi(x)$ is bounded, that is, $M:=\sup _{x \in \mathfrak{X}} \Psi(x)<+\infty$; and

(2) $\sum_{k} \Phi\left(a_{k}\right)<+\infty, a_{k} \in \mathfrak{X}$ implies $\sum_{k} \Psi\left(a_{k}\right)<+\infty$.

Then there exists $C>0$ such that

$$
\Psi(x) \leq C \Phi(x), \quad x \in \mathfrak{X} .
$$

Proof. For $x \in \mathfrak{X}$ satisfying $\Phi(x)=0$, we have $\Psi(x)=0$ by the second condition.

Define $\mathfrak{X}_{+}:=\{x \in \mathfrak{X} \mid \Phi(x)>0\}$ and assume

$$
C:=\sup _{x \in \mathfrak{X}_{+}} \frac{\Psi(x)}{\Phi(x)}=+\infty .
$$


Then for any $n \in \mathbb{N}$ there exists $x_{n} \in \mathfrak{X}_{+}$such that

$$
2^{n} M<\frac{\Psi\left(x_{n}\right)}{\Phi\left(x_{n}\right)} \leq \frac{M}{\Phi\left(x_{n}\right)},
$$

that is, $\Phi\left(x_{n}\right)<2^{-n}$. Define $r_{n}:=\left\langle\left\langle 2^{-n} \Phi\left(x_{n}\right)^{-1}\right\rangle\right.$ where $\left.\langle r\rangle\right\rangle$ denotes the greatest integer not greater than $r \in \mathbb{R}$.

Then we have

$$
1 \leq r_{n}=\left\langle 2^{-n} \Phi\left(x_{n}\right)^{-1}\right\rangle \leq 2^{-n} \Phi\left(x_{n}\right)^{-1}<r_{n}+1,
$$

so that $r_{n} \Phi\left(x_{n}\right) \leq 2^{-n}$.

Hence, for

$$
\left\{a_{m}\right\}:=(\overbrace{x_{1}, \ldots, x_{1}}^{r_{1}}, \overbrace{x_{2}, \ldots, x_{2}}^{r_{2}}, \ldots, \overbrace{x_{n}, \ldots, x_{n}}^{r_{2}}, \ldots) \in \mathfrak{X}_{+}^{\infty},
$$

we have

$$
\sum_{m} \Phi\left(a_{m}\right)=\sum_{n} r_{n} \Phi\left(x_{n}\right) \leq \sum_{n} 2^{-n}<+\infty .
$$

On the other hand, we have $2^{-(n+1)} \Phi\left(x_{n}\right)^{-1}<\left(r_{n}+1\right) / 2 \leq r_{n}$, and

$$
r_{n} \Psi\left(x_{n}\right)=r_{n} \frac{\Psi\left(x_{n}\right)}{\Phi\left(x_{n}\right)} \Phi\left(x_{n}\right) \geq 2^{-(n+1)} \Phi\left(x_{n}\right)^{-1} 2^{n} M \Phi\left(x_{n}\right)=\frac{M}{2} .
$$

Consequently, we have

$$
\sum_{m} \Psi\left(a_{m}\right)=\sum_{n} r_{n} \Psi\left(x_{n}\right) \geq \sum_{n} \frac{M}{2}=+\infty,
$$

which is a contradiction. Therefore, $C<+\infty$ and the lemma is proved.

Proof of Proposition 3. (QM1), (QM2) and (TRL) follow from the definition of $\gamma_{f}$.

We prove (QM3). Since $\Lambda_{2}^{0}(f)$ is a linear space, $\boldsymbol{a}=\left\{a_{k}\right\}, \boldsymbol{b}=\left\{b_{k}\right\} \in \Lambda_{2}^{0}(f)$ implies $\boldsymbol{a}-\boldsymbol{b} \in \Lambda_{2}^{0}(f)$. In other words,

$$
\sum_{k}\left[\xi_{f}\left(a_{k}\right)+\xi_{f}\left(b_{k}\right)\right]<+\infty, \quad a_{k}, b_{k} \in \mathbb{R}
$$

implies

$$
\sum_{k} \xi_{f}\left(a_{k}-b_{k}\right)<+\infty
$$

Since $\xi_{f}$ is bounded, applying Lemma 4 to $\Phi(u, v)=\xi_{f}(u)+\xi_{f}(v)$ and $\Psi(u, v)=$ $\xi_{f}(u-v),(u, v) \in \mathfrak{X}:=\mathbb{R}^{2}$, we have

$$
C:=\sup _{(u, v) \neq(0,0)} \frac{\xi_{f}(u-v)}{\xi_{f}(u)+\xi_{f}(v)}<+\infty,
$$

so that

$$
\begin{aligned}
\gamma_{f}(\boldsymbol{a}-\boldsymbol{b}, \mathbf{0}) & =\sqrt{\sum_{k} \xi_{f}\left(a_{k}-b_{k}\right)} \leq \sqrt{C}\left(\sqrt{\sum_{k} \xi_{f}\left(a_{k}\right)}+\sqrt{\sum_{k} \xi_{f}\left(b_{k}\right)}\right) \\
& =\sqrt{C}\left[\gamma_{f}(\boldsymbol{a}, \mathbf{0})+\gamma_{f}(\boldsymbol{b}, \mathbf{0})\right], \quad \boldsymbol{a}=\left\{a_{k}\right\}, \quad \boldsymbol{b}=\left\{b_{k}\right\} \in \Lambda_{2}^{0}(f),
\end{aligned}
$$

which proves (QM3) for $A:=\sqrt{C}$. 
LEMMA 5. For any natural number $N, \gamma_{f}$ satisfies

$$
\sup _{|t| \leq N} \gamma_{f}(t \boldsymbol{a}, \mathbf{0}) \leq \gamma_{f}(N \boldsymbol{a}, \mathbf{0}) \leq N A^{N-1} \gamma_{f}(\boldsymbol{a}, \mathbf{0}), \quad \boldsymbol{a} \in \Lambda_{2}^{0}(f),
$$

where A is given in the proof of Proposition 3.

Proof. Since $\gamma_{f}(t \boldsymbol{a}, \mathbf{0})$ is non-decreasing for $t \geq 0$ by the definition, we have the first inequality. The second inequality follows from (QM3).

THEOREM 6. For $f(\neq 0) \in L_{2}, \gamma_{f}$ is a translation invariant linear quasi-metric and $\left(\Lambda_{2}^{0}(f), \gamma_{f}\right)$ is a complete separable topological linear space.

Proof. We show $\left(\Lambda_{2}^{0}(f), \gamma_{f}\right)$ is a topological linear space. The continuity of the mapping

$$
(\boldsymbol{a}, \boldsymbol{b}) \rightarrow \boldsymbol{a}-\boldsymbol{b}:\left(\Lambda_{2}^{0}(f), \gamma_{f}\right) \times\left(\Lambda_{2}^{0}(f), \gamma_{f}\right) \rightarrow\left(\Lambda_{2}^{0}(f), \gamma_{f}\right)
$$

is derived from (QM3) and (TRL).

We show the continuity of the mapping

$$
(t, \boldsymbol{a}) \rightarrow \boldsymbol{t a}: \mathbb{R} \times\left(\Lambda_{2}^{0}(f), \gamma_{f}\right) \rightarrow\left(\Lambda_{2}^{0}(f), \gamma_{f}\right) .
$$

Since $\left(\Lambda_{2}^{0}(f), \gamma_{f}\right)$ satisfies the first axiom of countability, it is enough to show the sequential continuity.

For any fixed $\left(t_{0}, \boldsymbol{a}_{0}\right) \in \mathbb{R} \times \Lambda_{2}^{0}(f)$, assume $t_{n} \rightarrow t_{0}$ and $\boldsymbol{a}_{n} \rightarrow \boldsymbol{a}_{0}$. By (QM3) and (TRL) we have

$$
\gamma_{f}\left(t_{n} \boldsymbol{a}_{n}, t_{0} \boldsymbol{a}_{0}\right) \leq A\left[\gamma_{f}\left(t_{n}\left(\boldsymbol{a}_{n}-\boldsymbol{a}_{0}\right), \mathbf{0}\right)+\gamma_{f}\left(\left(t_{n}-t_{0}\right) \boldsymbol{a}_{0}, \mathbf{0}\right)\right] .
$$

Then we have $\gamma_{f}\left(\left(t_{n}-t_{0}\right) \boldsymbol{a}_{0}, \mathbf{0}\right) \rightarrow 0$ by Lemma 1 .

Since $t_{n} \rightarrow t_{0}$, without loss of generality, we may assume $\left|t_{n}\right|<N:=\left\langle\left|t_{0}\right|\right\rangle+2$. Then by Lemma 5 we have

$$
\gamma_{f}\left(t_{n}\left(\boldsymbol{a}_{n}-\boldsymbol{a}_{0}\right), \mathbf{0}\right) \leq N A^{N-1} \gamma_{f}\left(\boldsymbol{a}_{n}-\boldsymbol{a}_{0}, \mathbf{0}\right) \rightarrow 0 .
$$

Consequently we have $\gamma_{f}\left(t_{n} \boldsymbol{a}_{n}, t_{0} \boldsymbol{a}_{0}\right) \rightarrow 0$.

We show the completeness. Let $\boldsymbol{a}^{(m)}=\left\{a_{1}^{(m)}, a_{2}^{(m)}, \ldots, a_{k}^{(m)}, \ldots\right\} \in\left(\Lambda_{2}^{0}(f), \gamma_{f}\right)$ be a Cauchy sequence. For every $\varepsilon>0$ there exists a natural number $N$ such that for every $m, l \geq N$ we have

$$
\gamma_{f}\left(\boldsymbol{a}^{(m)}, \boldsymbol{a}^{(l)}\right)^{2}=\sum_{k} \xi_{f}\left(\left|a_{k}^{(m)}-a_{k}^{(l)}\right|\right)<\varepsilon^{2} .
$$

By the definition, $\xi_{f}(t) \rightarrow 0$ implies $t \rightarrow 0$, so that $\left\{a_{k}^{(m)}\right\}_{m=1}^{+\infty}, k=1,2, \ldots$, are Cauchy sequences in $\mathbb{R}$ and the limits $a_{k}:=\lim _{m \rightarrow+\infty} a_{k}^{(m)} \in \mathbb{R}$ exist. Since $\xi_{f}(t)$ is continuous, by Fatou's lemma we have for $\boldsymbol{a}:=\left\{a_{k}\right\} \in \mathbb{R}^{\infty}, m \geq N$,

$$
\begin{aligned}
\gamma_{f}\left(\boldsymbol{a}^{(m)}, \boldsymbol{a}\right)^{2} & =\sum_{k} \xi_{f}\left(\left|a_{k}^{(m)}-a_{k}\right|\right)=\sum_{k} \liminf _{l \rightarrow+\infty} \xi_{f}\left(\left|a_{k}^{(m)}-a_{k}^{(l)}\right|\right) \\
& \leq \liminf _{l \rightarrow+\infty} \sum_{k} \xi_{f}\left(\left|a_{k}^{(m)}-a_{k}^{(l)}\right|\right) \leq \varepsilon^{2},
\end{aligned}
$$

so that $\boldsymbol{a} \in \Lambda_{2}^{0}(f)$ and $\boldsymbol{a}^{(m)} \rightarrow \boldsymbol{a}$.

Let $\mathbb{Q}$ be the set of all rational numbers. Then the set

$$
\mathbb{Q}_{0}^{\infty}:=\left\{\left\{a_{n}\right\} \mid a_{n} \in \mathbb{Q} \text { and } a_{n}=0 \text { except for finitely many } n\right\}
$$

is dense in $\left(\Lambda_{2}^{0}(f), \gamma_{f}\right)$. Therefore $\left(\Lambda_{2}^{0}(f), \gamma_{f}\right)$ is separable. 
LEMMA 7. For any $t,|t| \leq 1$ and $\boldsymbol{a} \in \mathbb{R}^{\infty}$, we have

$$
d_{2}^{f}(t \boldsymbol{a}, \mathbf{0})^{2} \leq 16 \gamma_{f}(\boldsymbol{a}, \mathbf{0})^{2} \leq 9 \int_{-1}^{1} d_{2}^{f}(s \boldsymbol{a}, \mathbf{0})^{2} d s
$$

Proof. The first inequality is derived from ( $\boldsymbol{\Xi})$ as follows. For $\boldsymbol{a}=\left\{a_{k}\right\} \in \mathbb{R}^{\infty}$, we have

$$
\begin{aligned}
\frac{1}{2} d_{2}^{f}(t \boldsymbol{a}, \mathbf{0})^{2} & =\sum_{k}\left(\int_{\left|a_{k} \alpha\right| \leq 1}+\int_{\left|a_{k} \alpha\right|>1}\right)\left(1-\cos t a_{k} \alpha\right)|\hat{f}(\alpha)|^{2} d \alpha \\
& \leq \sum_{k} a_{k}^{2} \int_{0}^{1 /\left|a_{k}\right|} \alpha^{2}|\hat{f}(\alpha)|^{2} d \alpha+4 \sum_{k} \int_{1 /\left|a_{k}\right|}^{+\infty}|\hat{f}(\alpha)|^{2} d \alpha \\
& \leq 8 \gamma_{f}(\boldsymbol{a}, \mathbf{0})^{2} .
\end{aligned}
$$

To prove the second inequality, assume $\int_{-1}^{1} d_{2}^{f}(s \boldsymbol{a}, \mathbf{0})^{2} d s<+\infty$. Then, since we have $d_{2}^{f}(s \boldsymbol{a}, \mathbf{0})<+\infty \lambda$-almost everywhere on $[-1,1]$, where $\lambda$ is the Lebesgue measure, there exists $r>0$ such that the set $P:=\left\{s \in[-1,1] \mid d_{2}^{f}(s \boldsymbol{a}, \mathbf{0}) \leq r\right\}$ has a positive measure. By the continuity of the function

$$
s \rightarrow \lambda((P-s) \ominus P)=\int_{-\infty}^{+\infty}\left|I_{P}(x+s)-I_{P}(x)\right|^{2} d x,
$$

where $\ominus$ is the symmetric difference and $I_{P}(x)$ is the indicator function of $P$, there exists a neighborhood $V$ of 0 such that

$$
\lambda((P-s) \ominus P)<\lambda(P) \text { for } s \in V .
$$

Then for every $s \in V$, we have $(P-s) \cap P \neq \emptyset$ and there exist $p_{1}, p_{2} \in P$ such that $s=p_{1}-p_{2}$. Therefore, we have

$$
d_{2}^{f}(s \boldsymbol{a}, \mathbf{0}) \leq d_{2}^{f}\left(p_{1} \boldsymbol{a}, \mathbf{0}\right)+d_{2}^{f}\left(p_{2} \boldsymbol{a}, \mathbf{0}\right) \leq 2 r .
$$

Let $N$ be a natural number satisfying $[-1,1] \subset N V$. Then we have

$$
\sup _{|t| \leq 1} d_{2}^{f}(t \boldsymbol{a}, \mathbf{0}) \leq N \sup _{u \in V} d_{2}^{f}(u \boldsymbol{a}, \mathbf{0}) \leq 2 N r .
$$

We have proved

$$
\sum_{k} \int_{-\infty}^{+\infty}\left(1-\cos t a_{k} \alpha\right)|\hat{f}(\alpha)|^{2} d \alpha=\frac{1}{2} d_{2}^{f}(t \boldsymbol{a}, \mathbf{0})^{2} \leq 2(N r)^{2} \quad \text { for }|t| \leq 1
$$


Taking the integral $(1 / 2) \int_{-1}^{1} d t$ in both sides we have

$$
\begin{aligned}
2(N r)^{2} \geq & \frac{1}{4} \int_{-1}^{1} d_{2}^{f}(t \boldsymbol{a}, \mathbf{0})^{2} d t \\
\geq & \sum_{k} \frac{1}{2} \int_{-1}^{1} d t \frac{1}{3} \int_{\left|a_{k} \alpha\right| \leq 1} t^{2} a_{k}^{2} \alpha^{2}|\hat{f}(\alpha)|^{2} d \alpha \\
& +\sum_{k} \int_{\left|a_{k} \alpha\right|>1}\left(1-\frac{\sin a_{k} \alpha}{a_{k} \alpha}\right)|\hat{f}(\alpha)|^{2} d \alpha \\
\geq & \frac{1}{9} \sum_{k} a_{k}^{2} \int_{\left|a_{k} \alpha\right| \leq 1} \alpha^{2}|\hat{f}(\alpha)|^{2} d \alpha+(1-\sin 1) \sum_{k} \int_{\left|a_{k} \alpha\right|>1}|\hat{f}(\alpha)|^{2} d \alpha \\
\geq & \frac{2}{9} \sum_{k} a_{k}^{2} \int_{0}^{1 /\left|a_{k}\right|} \alpha^{2}|\hat{f}(\alpha)|^{2} d \alpha+\frac{2}{9} \sum_{k} \int_{1 /\left|a_{k}\right|}^{+\infty}|\hat{f}(\alpha)|^{2} d \alpha=\frac{4}{9} \gamma_{f}(\boldsymbol{a}, \mathbf{0})^{2},
\end{aligned}
$$

which proves the second inequality by $1 / 9<1-\sin 1$ and by $(\boldsymbol{\Xi})$.

COROLlaRY 8. For $f(\neq 0) \in L_{2}$, the inclusion $\Lambda_{2}^{0}(f) \subset \Lambda_{2}(f)$ is continuous.

THEOREM 9. For $f(\neq 0) \in L_{2}, \gamma_{f}(\boldsymbol{a}, \boldsymbol{b})$ and the metric $\sup _{|t| \leq 1} d_{2}^{f}($ ta, t $\boldsymbol{b})$ are equivalent on $\Lambda_{2}^{0}(f)$.

Proof. Assume $\boldsymbol{a} \in \Lambda_{2}^{0}(f)$, that is, $\gamma_{f}(\boldsymbol{a}, \mathbf{0})<+\infty$. Then by Lemma 7 we have

$$
\frac{2 \sqrt{2}}{3} \gamma_{f}(\boldsymbol{a}, \mathbf{0}) \leq \sqrt{\frac{1}{2} \int_{-1}^{1} d_{2}^{f}(t \boldsymbol{a}, \mathbf{0})^{2} d t} \leq \sup _{|t| \leq 1} d_{2}^{f}(t \boldsymbol{a}, \mathbf{0}) \leq 4 \gamma_{f}(\boldsymbol{a}, \mathbf{0}), \quad \boldsymbol{a} \in \Lambda_{2}^{0}(f) .
$$

THEOREM 10. For $f(\neq 0) \in L_{2}$, the following two conditions are equivalent:

(i) $\Lambda_{2}(f)$ is a linear space;

(ii) $\gamma_{f}$ is equivalent to $d_{2}^{f}$ on $\Lambda_{2}^{0}(f)$.

Proof. Assume (i). Then by Lemma 2 we have $\Lambda_{2}(f) \subset \Lambda_{2}^{0}(f)$, that is, for $\boldsymbol{a}=\left\{a_{k}\right\} \in$ $\Lambda_{2}(f)$,

$$
d_{2}^{f}(\boldsymbol{a}, \mathbf{0})^{2}=\sum_{k} \int_{-\infty}^{+\infty}\left|f\left(x-a_{k}\right)-f(x)\right|^{2} d x<+\infty, \quad a_{k} \in \mathbb{R}
$$

implies

$$
\gamma_{f}(\boldsymbol{a}, \mathbf{0})^{2}=\sum_{k} \xi_{f}\left(a_{k}\right)<+\infty
$$

Applying Lemma 4 to $\Phi(u)=\int_{-\infty}^{+\infty}|f(x-u)-f(x)|^{2} d x, \Psi(u)=\xi_{f}(u), u \in \mathfrak{X}:=\mathbb{R}$, we have for a constant $C>0$

$$
\xi_{f}(u) \leq C \int_{-\infty}^{+\infty}|f(x-u)-f(x)|^{2} d x, \quad u \in \mathbb{R},
$$

which implies $\gamma_{f}(\boldsymbol{a}, \boldsymbol{b}) \leq \sqrt{C} d_{2}^{f}(\boldsymbol{a}, \boldsymbol{b})$. On the other hand, by Lemma 7 we have $d_{2}^{f}(\boldsymbol{a}, \boldsymbol{b}) \leq$ $4 \gamma_{f}(\boldsymbol{a}, \boldsymbol{b})$, which implies (ii).

Conversely, assume (ii). Then there exists $B \geq 1$ such that

$$
\gamma_{f}(\boldsymbol{a}, \mathbf{0}) \leq B d_{2}^{f}(\boldsymbol{a}, \mathbf{0}), \quad \boldsymbol{a} \in \Lambda_{2}^{0}(f) .
$$


We show $\Lambda_{2}(f)=\Lambda_{2}^{0}(f)$ which implies the linearity of $\Lambda_{2}(f)$ by Lemma 2 . For any $\boldsymbol{b}=\left\{b_{k}\right\} \in \Lambda_{2}(f)$, set $\boldsymbol{b}_{n}:=\left\{b_{1}, b_{2}, \ldots, b_{n}, 0,0, \ldots\right\}$. Then $\boldsymbol{b}_{n} \in \Lambda_{2}^{0}(f)$ and

$$
\gamma_{f}(\boldsymbol{b}, \mathbf{0})=\lim _{n \rightarrow+\infty} \gamma_{f}\left(\boldsymbol{b}_{n}, \mathbf{0}\right) \leq B \lim _{n \rightarrow+\infty} d_{2}^{f}\left(\boldsymbol{b}_{n}, \mathbf{0}\right) \leq B d_{2}^{f}(\boldsymbol{b}, \mathbf{0})<+\infty,
$$

which implies $\boldsymbol{b} \in \Lambda_{2}^{0}(f)$ and $\Lambda_{2}(f) \subset \Lambda_{2}^{0}(f)$. The inclusion $\Lambda_{2}^{0}(f) \subset \Lambda_{2}(f)$ is proved in Lemma 2, so that we have $\Lambda_{2}(f)=\Lambda_{2}^{0}(f)$.

By Theorem 10, in the case where $\Lambda_{2}(f)$ is a linear space, $\left(\Lambda_{2}^{0}(f), \gamma_{f}\right)$ is a visible sequential representation of $\left(\Lambda_{2}(f), d_{2}^{f}\right)$.

\section{Outer approximation $\left(\Lambda_{2}^{\varphi}(f), \delta_{f}\right)$}

In this section, we discuss the linearity of the outer approximation $\left(\Lambda_{2}^{\varphi}(f), \delta_{f}\right)$ for $f(\neq 0) \in L_{2}$.

For $L>0$ define

$$
\begin{gathered}
\delta_{f}^{L}(\boldsymbol{a}, \boldsymbol{b}):=\sqrt{\sum_{k}\left[\left|a_{k}-b_{k}\right| \wedge \frac{1}{L}\right]^{2}\left[1+\varphi_{f}\left(\frac{1}{\left|a_{k}-b_{k}\right| \wedge(1 / L)}\right)\right]} \\
\boldsymbol{a}=\left\{a_{k}\right\}, \boldsymbol{b}=\left\{b_{k}\right\} \in \mathbb{R}^{\infty} .
\end{gathered}
$$

LEMMA 11. For $L>0$ there exist $m(L), M(L)>0$ such that

$$
m(L) \delta_{f}^{L}(\boldsymbol{a}, \boldsymbol{b}) \leq \delta_{f}(\boldsymbol{a}, \boldsymbol{b}) \leq M(L) \delta_{f}^{L}(\boldsymbol{a}, \boldsymbol{b}), \quad \boldsymbol{a}, \boldsymbol{b} \in \mathbb{R}^{\infty} .
$$

Proof. By elementary estimations we have for $x \in \mathbb{R}, 0<L<L^{\prime}<+\infty$,

$$
\begin{aligned}
& \frac{1}{K\left(L, L^{\prime}\right)}\left(|x| \wedge \frac{1}{L^{\prime}}\right)^{2}\left[1+\varphi_{f}\left(\frac{1}{|x| \wedge\left(1 / L^{\prime}\right)}\right)\right] \\
& \quad \leq\left(|x| \wedge \frac{1}{L}\right)^{2}\left[1+\varphi_{f}\left(\frac{1}{|x| \wedge(1 / L)}\right)\right] \\
& \quad \leq K\left(L, L^{\prime}\right)\left(|x| \wedge \frac{1}{L^{\prime}}\right)^{2}\left[1+\varphi_{f}\left(\frac{1}{|x| \wedge\left(1 / L^{\prime}\right)}\right)\right],
\end{aligned}
$$

where $K\left(L, L^{\prime}\right):=\left(L^{\prime} / L\right)^{2}\left(1+\varphi_{f}\left(L^{\prime}\right)\right) /\left(1+\varphi_{f}(L)\right)$.

By Lemma 11, for any $L>0$ we have $\Lambda_{2}^{\varphi}(f)=\left\{\boldsymbol{a} \in \mathbb{R}^{\infty} \mid \delta_{f}^{L}(\boldsymbol{a}, \mathbf{0})<+\infty\right\}$.

THEOREM 12. If $H\left(\varphi_{f}\right)<+\infty$, then for every $\boldsymbol{a} \in \Lambda_{2}^{\varphi}(f)$, the segment $[-1,1] \boldsymbol{a}:=\{$ t $\boldsymbol{a} \mid$ $-1 \leq t \leq 1\}$ is contained in $\Lambda_{2}^{\varphi}(f)$.

Proof. Assume $H\left(\varphi_{f}\right)<+\infty$. Then there exists $h \geq 2$ such that $D\left(h ; \varphi_{f}\right)<+\infty$. Therefore, the theorem is proved by the following lemma.

LEMMA 13. For $f(\neq 0) \in L_{2}$ and $h \geq 2$, we have $D\left(h ; \varphi_{f}\right)<+\infty$ if and only if there exists $F \geq 1$ such that

$$
\delta_{f}(t \boldsymbol{a}, \mathbf{0}) \leq F t^{1-(h / 2)} \delta_{f}(\boldsymbol{a}, \mathbf{0}), \quad \boldsymbol{a} \in \Lambda_{2}^{\varphi}(f), 0<t \leq 1 .
$$


Proof. Assume $D\left(h ; \varphi_{f}\right)<+\infty$ for $h \geq 2$, which is equivalent to $D\left(h ; 1+\varphi_{f}\right)<+\infty$. Then by

$$
\begin{aligned}
D\left(h ; 1+\varphi_{f}\right)= & \limsup _{\substack{x \rightarrow+\infty \\
\varphi_{f}(x)>0}} \sup _{T \geq 1} \frac{1+\varphi_{f}(T x)}{\left(1+\varphi_{f}(x)\right) T^{h}} \\
= & \limsup _{\substack{x \downarrow 0 \\
\varphi_{f}(1 / x)>0}} \sup _{0<t \leq 1} \frac{x^{2} t^{2}\left[1+\varphi_{f}(1 /(t x))\right]}{x^{2}\left(1+\varphi_{f}(1 / x)\right) t^{2-h}}<+\infty,
\end{aligned}
$$

there exist $K>0$ and $L>1$ such that

$$
(t x)^{2}\left[1+\varphi_{f}\left(\frac{1}{t x}\right)\right] \leq K t^{2-h} x^{2}\left[1+\varphi_{f}\left(\frac{1}{x}\right)\right], \quad 0<t \leq 1,0<x \leq \frac{1}{L} .
$$

Applying the above to $x=\left|a_{k}\right| \wedge(1 / L)$ and $t:=\left[\left(s\left|a_{k}\right|\right) \wedge(1 / L)\right] /\left[\left|a_{k}\right| \wedge(1 / L)\right] \in$ $(0,1], 0<s \leq 1$, we obtain

$$
\begin{aligned}
& \left(\left(s\left|a_{k}\right|\right) \wedge \frac{1}{L}\right)^{2}\left[1+\varphi_{f}\left(\frac{1}{\left(s\left|a_{k}\right|\right) \wedge(1 / L)}\right)\right] \\
& \leq K s^{2-h}\left(\left|a_{k}\right| \wedge \frac{1}{L}\right)^{2}\left[1+\varphi_{f}\left(\frac{1}{\left|a_{k}\right| \wedge(1 / L)}\right)\right]
\end{aligned}
$$

which means

$$
\delta_{f}^{L}(s \boldsymbol{a}, \mathbf{0}) \leq \sqrt{K} s^{1-(h / 2)} \delta_{f}^{L}(\boldsymbol{a}, \mathbf{0}), \quad \boldsymbol{a} \in \Lambda_{2}^{\varphi}(f) .
$$

By Lemma 11, there exist $m(L), M(L)>0$ such that

$$
m(L) \delta_{f}^{L}(\boldsymbol{a}, \boldsymbol{b}) \leq \delta_{f}(\boldsymbol{a}, \boldsymbol{b}) \leq M(L) \delta_{f}^{L}(\boldsymbol{a}, \boldsymbol{b}), \quad \boldsymbol{a}, \boldsymbol{b} \in \mathbb{R}^{\infty},
$$

and we have for $F:=(M(L) / m(L)) \sqrt{K}$,

$$
\delta_{f}(t \boldsymbol{a}, \mathbf{0}) \leq F t^{1-(h / 2)} \delta_{f}(\boldsymbol{a}, \mathbf{0}), \quad 0<t \leq 1, \boldsymbol{a} \in \Lambda_{2}^{\varphi}(f) .
$$

Conversely, assume $\delta_{f}(t \boldsymbol{a}, \mathbf{0}) \leq F t^{1-(h / 2)} \delta_{f}(\boldsymbol{a}, \mathbf{0}), \quad \boldsymbol{a} \in \Lambda_{2}^{\varphi}(f), \quad 0<t \leq 1$. Then for $\boldsymbol{a}=\{u, 0,0, \ldots\}, u \in(0,1)$, we have

$$
(t u)^{2}\left[1+\varphi_{f}\left(\frac{1}{t u}\right)\right] \leq F^{2} t^{2-h} u^{2}\left[1+\varphi_{f}\left(\frac{1}{u}\right)\right], \quad 0<t \leq 1,
$$

so that

$$
\frac{1+\varphi_{f}(T x)}{\left[1+\varphi_{f}(x)\right] T^{h}} \leq F^{2}, \quad x:=\frac{1}{u} \geq 1, T:=\frac{1}{t} \geq 1,
$$

which implies $D\left(h ; 1+\varphi_{f}\right)<+\infty$ and $D\left(h ; \varphi_{f}\right)<+\infty$.

COROLlaRY 14. For $f(\neq 0) \in L_{2}$, we have $D\left(2 ; \varphi_{f}\right)<+\infty$ if and only if for a constant $F \geq 1$,

$$
\sup _{|t| \leq 1} \delta_{f}(t \boldsymbol{a}, \mathbf{0}) \leq F \delta_{f}(\boldsymbol{a}, \mathbf{0}), \quad \boldsymbol{a} \in \Lambda_{2}^{\varphi}(f)
$$

In particular, if $D\left(2 ; \varphi_{f}\right)<+\infty$, then $[-1,1] \boldsymbol{a} \subset \Lambda_{2}^{\varphi}(f)$ for any $\boldsymbol{a} \in \Lambda_{2}^{\varphi}(f)$.

Proof. Since $\delta_{f}(-\boldsymbol{a}, \mathbf{0})=\delta_{f}(\boldsymbol{a}, \mathbf{0})$, the results follow from Lemma 13. 
LEMma 15. For $f(\neq 0) \in L_{2}$, define

$$
\zeta_{f}(t):= \begin{cases}(|t| \wedge 1)^{2}\left[1+\varphi_{f}\left(\frac{1}{|t| \wedge 1}\right)\right], & t \neq 0, \\ 0, & t=0 .\end{cases}
$$

Then $\zeta_{f}(t)$ is a bounded continuous function on $\mathbb{R}$.

Proof. We have $\lim _{t \rightarrow 0} \zeta_{f}(t)=0$ (see [3, Lemma 4.4]), which proves the lemma.

THEOREM 16. For $f(\neq 0) \in L_{2}$, if $\Lambda_{2}^{\varphi}(f)$ is a linear space, then we have $D\left(2 ; \varphi_{f}\right)<+\infty$.

Proof. Assume that $\Lambda_{2}^{\varphi}(f)$ is a linear space. Then $\boldsymbol{a}=\left\{a_{k}\right\}, \boldsymbol{b}=\left\{b_{k}\right\} \in \Lambda_{2}^{\varphi}(f)$ implies $\boldsymbol{a}+\boldsymbol{b} \in \Lambda_{2}^{\varphi}(f)$. In other words,

$$
\sum_{k}\left[\zeta_{f}\left(a_{k}\right)+\zeta_{f}\left(b_{k}\right)\right]<+\infty, \quad a_{k}, b_{k} \in \mathbb{R}
$$

implies

$$
\sum_{k} \zeta_{f}\left(a_{k}+b_{k}\right)<+\infty
$$

Since $\zeta_{f}(x)$ is bounded, Lemma 4 is applicable to $\Phi(u, v)=\zeta_{f}(u)+\zeta_{f}(v)$ and $\Psi(u, v)=$ $\zeta_{f}(u+v), x=(u, v) \in \mathfrak{X}:=\mathbb{R}^{2}$. We have for a constant $C \geq 1$,

$$
\zeta_{f}(a+b) \leq C\left[\zeta_{f}(a)+\zeta_{f}(b)\right], \quad a, b \in \mathbb{R},
$$

and

$$
\delta_{f}(\boldsymbol{a}+\boldsymbol{b}, \mathbf{0}) \leq \sqrt{C}\left[\delta_{f}(\boldsymbol{a}, \mathbf{0})+\delta_{f}(\boldsymbol{b}, \mathbf{0})\right], \quad \boldsymbol{a}, \boldsymbol{b} \in \Lambda_{2}^{\varphi}(f) .
$$

By the linearity of $\Lambda_{2}^{\varphi}(f)$, for any fixed $t \in \mathbb{R}, \delta_{f}(\boldsymbol{a}, \boldsymbol{0})^{2}<+\infty$ implies $\delta_{f}(t \boldsymbol{a}, \boldsymbol{0})^{2}<$ $+\infty$, that is,

$$
\sum_{k} \zeta_{f}\left(a_{k}\right)<+\infty, \quad a_{k} \in \mathbb{R}
$$

implies

$$
\sum_{k} \zeta_{f}\left(t a_{k}\right)<+\infty
$$

Applying Lemma 4 to $\Phi(u)=\zeta_{f}(u)$ and $\Psi(u)=\zeta_{f}(t u), u \in \mathfrak{X}:=\mathbb{R}$. We have

$$
C(t):=\sup _{u \neq 0} \frac{\zeta_{f}(t u)}{\zeta_{f}(u)}<+\infty,
$$

and

$$
\delta_{f}(t \boldsymbol{a}, \mathbf{0}) \leq \sqrt{C(t)} \delta_{f}(\boldsymbol{a}, \mathbf{0}), \quad \boldsymbol{a} \in \Lambda_{2}^{\varphi}(f) .
$$

We show that for a constant $F \geq 1$,

$$
\sup _{|t| \leq 1} \delta_{f}(t \boldsymbol{a}, \mathbf{0}) \leq F \delta_{f}(\boldsymbol{a}, \mathbf{0}), \quad \boldsymbol{a} \in \Lambda_{2}^{\varphi}(f) .
$$

By Lemma $15 \sqrt{C(t)}$ is lower semi-continuous. By the Baire category theorem there exists a natural number $N$ such that $U:=\{t \in \mathbb{R} \mid \sqrt{C(t)} \leq N\}$ contains an interior point, so that $W:=U-U$ is a neighborhood of 0 . Remark that $C(t)=C(-t)$ since $\zeta_{f}(t)=\zeta_{f}(-t)$. 
For any $w=u-v \in W, u, v \in U$, we have

$$
\begin{aligned}
\delta_{f}(w \boldsymbol{a}, \mathbf{0}) & \leq \sqrt{C}\left[\delta_{f}(u \boldsymbol{a}, \mathbf{0})+\delta_{f}(-v \boldsymbol{a}, \mathbf{0})\right] \\
& \leq \sqrt{C}\left[\sqrt{C(u)} \delta_{f}(\boldsymbol{a}, \mathbf{0})+\sqrt{C(-v)} \delta_{f}(\boldsymbol{a}, \mathbf{0})\right] \leq 2 \sqrt{C} N \delta_{f}(\boldsymbol{a}, \mathbf{0}) .
\end{aligned}
$$

Let $n$ be a natural number satisfying $[-1,1] \subset n W$, then for every $t \in[-1,1]$ and for every $\boldsymbol{a} \in \Lambda_{2}^{\varphi}(f)$ we have

$$
\begin{aligned}
\delta_{f}(t \boldsymbol{a}, \mathbf{0}) & =\delta_{f}(\overbrace{\frac{t}{n} \boldsymbol{a}+\cdots+\frac{t}{n} \boldsymbol{a}}^{n}, \mathbf{0}) \leq \sqrt{C}^{n-1} n \delta_{f}\left(\frac{t}{n} \boldsymbol{a}, \mathbf{0}\right) \\
& \leq 2 n \sqrt{C}^{n} N \delta_{f}(\boldsymbol{a}, \mathbf{0}),
\end{aligned}
$$

so that for $F:=2 n \sqrt{C}^{n} N$, we prove the required.

By Corollary 14 we have $D\left(2 ; \varphi_{f}\right)<+\infty$.

Lemma 17. For $f(\neq 0) \in L_{2}$ assume $D\left(2 ; \varphi_{f}\right)<+\infty$. Then there exists $A \geq 1$ such that

$$
\delta_{f}(\boldsymbol{a}+\boldsymbol{b}, \mathbf{0}) \leq A\left[\delta_{f}(\boldsymbol{a}, \mathbf{0})+\delta_{f}(\boldsymbol{b}, \mathbf{0})\right], \quad \boldsymbol{a}, \boldsymbol{b} \in \Lambda_{2}^{\varphi}(f) .
$$

Proof. Assume $D\left(2 ; \varphi_{f}\right)<+\infty$. Then we have $D\left(2 ; 1+\varphi_{f}\right)<+\infty$, so that there exist $1 \leq K, L<+\infty$ satisfying $1+\varphi_{f}(T x) \leq K T^{2}\left(1+\varphi_{f}(x)\right), x \geq L, T \geq 1$. For any $u, v>$ 0 satisfying $u \leq v \leq 1 / L$, putting $x=1 / v, T=v / u \geq 1$, we have

$$
u^{2}\left[1+\varphi_{f}\left(\frac{1}{u}\right)\right]=u^{2}\left[1+\varphi_{f}\left(\frac{v}{u} \frac{1}{v}\right)\right] \leq K v^{2}\left[1+\varphi_{f}\left(\frac{1}{v}\right)\right] .
$$

For $u=\left|a_{k}+b_{k}\right| \wedge\{1 /(2 L)\}, \quad v=\left[\left|a_{k}\right| \wedge\{1 /(2 L)\}\right]+\left[\left|b_{k}\right| \wedge\{1 /(2 L)\}\right]$ and $T:=$ $v / u \geq 1$ we have

$$
\begin{aligned}
& \left(\left|a_{k}+b_{k}\right| \wedge \frac{1}{2 L}\right)^{2}\left[1+\varphi_{f}\left(\frac{1}{\left|a_{k}+b_{k}\right| \wedge\{1 /(2 L)\}}\right)\right] \\
& \leq K\left[\left(\left|a_{k}\right| \wedge \frac{1}{2 L}\right)+\left(\left|b_{k}\right| \wedge \frac{1}{2 L}\right)\right]^{2}\left[1+\varphi_{f}\left(\frac{1}{\left[\left|a_{k}\right| \wedge\{1 /(2 L)\}\right]+\left[\left|b_{k}\right| \wedge\{1 /(2 L)\}\right]}\right)\right] \\
& \leq 2 K\left\{\left(\left|a_{k}\right| \wedge \frac{1}{2 L}\right)^{2}\left[1+\varphi_{f}\left(\frac{1}{\left|a_{k}\right| \wedge\{1 /(2 L)\}}\right)\right]\right. \\
& \left.\quad+\left(\left|b_{k}\right| \wedge \frac{1}{2 L}\right)^{2}\left[1+\varphi_{f}\left(\frac{1}{\left|b_{k}\right| \wedge\{1 /(2 L)\}}\right)\right]\right\} .
\end{aligned}
$$

Therefore, we have

$$
\delta_{f}^{2 L}(\boldsymbol{a}+\boldsymbol{b}, \mathbf{0}) \leq \sqrt{2 K}\left[\delta_{f}^{2 L}(\boldsymbol{a}, \mathbf{0})+\delta_{f}^{2 L}(\boldsymbol{b}, \mathbf{0})\right], \quad \boldsymbol{a}, \boldsymbol{b} \in \Lambda_{2}^{\varphi}(f),
$$

and Lemma 11 implies the required result.

Lemma 18. For $f(\neq 0) \in L_{2}$, if $D\left(2 ; \varphi_{f}\right)<+\infty$ then $\Lambda_{2}^{\varphi}(f)$ is a linear space and $\delta_{f}$ is a translation invariant quasi-metric on $\Lambda_{2}^{\varphi}(f)$. 
Proof. By Lemma 17, $\delta_{f}$ satisfies (QM3) and $\Lambda_{2}^{\varphi}(f)$ is an additive group. By Corollary 14 $\Lambda_{2}^{\varphi}(f)$ is closed under the scalar multiplication $t \boldsymbol{a}$ for $t \in[-1,1], \boldsymbol{a} \in \Lambda_{2}^{\varphi}(f)$, so that $\Lambda_{2}^{\varphi}(f)$ is a linear space. (QM1), (QM2) and (TRL) follow from the definition of $\delta_{f}$.

LeMma 19. For $f(\neq 0) \in L_{2}$, assume $D\left(2 ; \varphi_{f}\right)<+\infty$. Then for any $\boldsymbol{a} \in \Lambda_{2}^{\varphi}(f), \delta_{f}($ ta, $\mathbf{0})$ is continuous in $t \in[-1,1]$.

Proof. For any $\boldsymbol{a}=\left\{a_{k}\right\} \in \Lambda_{2}^{\varphi}(f)$, by Corollary 14 we have for a constant $F \geq 1$,

$$
\delta_{f}(t \boldsymbol{a}, \mathbf{0})^{2}=\sum_{k} \zeta_{f}\left(t a_{k}\right) \leq F^{2} \sum_{k} \zeta_{f}\left(a_{k}\right)=F^{2} \delta_{f}(\boldsymbol{a}, \mathbf{0})^{2}<+\infty, \quad t \in[-1,1]
$$

and by the dominated convergence theorem, $\delta_{f}(t \boldsymbol{a}, \mathbf{0})$ is continuous in $t \in[-1,1]$.

Theorem 20. For $f(\neq 0) \in L_{2}$, assume $D\left(2 ; \varphi_{f}\right)<+\infty$. Then $\delta_{f}$ is a translation invariant linear quasi-metric which makes $\left(\Lambda_{2}^{\varphi}(f), \delta_{f}\right)$ a complete separable topological linear space.

Proof. We show $\left(\Lambda_{2}^{\varphi}(f), \delta_{f}\right)$ is a topological linear space. The continuity of the mapping

$$
(\boldsymbol{a}, \boldsymbol{b}) \rightarrow \boldsymbol{a}-\boldsymbol{b}:\left(\Lambda_{2}^{\varphi}(f), \delta_{f}\right) \times\left(\Lambda_{2}^{\varphi}(f), \delta_{f}\right) \rightarrow\left(\Lambda_{2}^{\varphi}(f), \delta_{f}\right)
$$

is derived from (QM3) and (TRL).

We show the continuity of the mapping

$$
(t, \boldsymbol{a}) \rightarrow t \boldsymbol{a}: \mathbb{R} \times\left(\Lambda_{2}^{\varphi}(f), \delta_{f}\right) \rightarrow\left(\Lambda_{2}^{\varphi}(f), \delta_{f}\right) .
$$

Since $\left(\Lambda_{2}^{\varphi}(f), \delta_{f}\right)$ satisfies the first axiom of countability, we show the sequential continuity. For any fixed $\left(t_{0}, \boldsymbol{a}_{0}\right) \in \mathbb{R} \times \Lambda_{2}^{\varphi}(f)$, assume $t_{n} \rightarrow t_{0}$ and $\boldsymbol{a}_{n} \rightarrow \boldsymbol{a}_{0}$. By (QM3) and (TRL) we have

$$
\delta_{f}\left(t_{n} \boldsymbol{a}_{n}, t_{0} \boldsymbol{a}_{0}\right) \leq A\left[\delta_{f}\left(t_{n}\left(\boldsymbol{a}_{n}-\boldsymbol{a}_{0}\right), \mathbf{0}\right)+\delta_{f}\left(\left(t_{n}-t_{0}\right) \boldsymbol{a}_{0}, \mathbf{0}\right)\right] .
$$

Then we have $\delta_{f}\left(\left(t_{n}-t_{0}\right) \boldsymbol{a}_{0}, \mathbf{0}\right) \rightarrow 0$ by Lemma 19 .

Since $t_{n} \rightarrow t_{0}$, without loss of generality, we may assume $\left|t_{n}\right|<N:=\left\langle\left|t_{0}\right|\right\rangle+2$. Then by (QM3) and Corollary 14, we have

$$
\begin{aligned}
\delta_{f}\left(t_{n}\left(\boldsymbol{a}_{n}-\boldsymbol{a}_{0}\right), \boldsymbol{0}\right) & =\delta_{f}(\overbrace{\frac{t_{n}}{N}\left(\boldsymbol{a}_{n}-\boldsymbol{a}_{0}\right)+\cdots+\frac{t_{n}}{N}\left(\boldsymbol{a}_{n}-\boldsymbol{a}_{0}\right)}^{N}, \mathbf{0}) \\
& \leq N A^{N-1} \delta_{f}\left(\frac{t_{n}}{N}\left(\boldsymbol{a}_{n}-\boldsymbol{a}_{0}\right), \mathbf{0}\right) \\
& \leq N A^{N-1} F \delta_{f}\left(\boldsymbol{a}_{n}-\boldsymbol{a}_{0}, \mathbf{0}\right) \rightarrow 0 .
\end{aligned}
$$

Consequently, we have $\delta_{f}\left(t_{n} \boldsymbol{a}_{n}, t_{0} \boldsymbol{a}_{0}\right) \rightarrow 0$.

We show the completeness. Let $\boldsymbol{a}^{(m)}:=\left\{a_{1}^{(m)}, a_{2}^{(m)}, \ldots, a_{k}^{(m)}, \ldots\right\}$ be a Cauchy sequence in $\left(\Lambda_{2}^{\varphi}(f), \delta_{f}\right)$. For every $\varepsilon>0$ there exists a natural number $N$ such that for every $m, l \geq N$ we have

$$
\delta_{f}\left(\boldsymbol{a}^{(m)}, \boldsymbol{a}^{(l)}\right)^{2}=\sum_{k} \zeta_{f}\left(a_{k}^{(m)}-a_{k}^{(l)}\right)<\varepsilon^{2} .
$$

Since $\zeta_{f}(t) \rightarrow 0$ implies $t \rightarrow 0,\left\{a_{k}^{(m)}\right\}_{m=1}^{+\infty}, k=1,2, \ldots$, are Cauchy sequences in $\mathbb{R}$ and the limits $a_{k}:=\lim _{m \rightarrow+\infty} a_{k}^{(m)} \in \mathbb{R}$ exist. Since $\zeta_{f}(t)$ is bounded and continuous by Lemma 15 , 
by Fatou's lemma we have for $\boldsymbol{a}:=\left\{a_{k}\right\} \in \mathbb{R}^{\infty}, m \geq N$,

$$
\begin{aligned}
\delta_{f}\left(\boldsymbol{a}^{(m)}, \boldsymbol{a}\right)^{2} & =\sum_{k} \zeta_{f}\left(\left|a_{k}^{(m)}-a_{k}\right|\right)=\sum_{k} \liminf _{l \rightarrow+\infty} \zeta_{f}\left(\left|a_{k}^{(m)}-a_{k}^{(l)}\right|\right) \\
& \leq \liminf _{l \rightarrow+\infty} \sum_{k} \zeta_{f}\left(\left|a_{k}^{(m)}-a_{k}^{(l)}\right|\right) \leq \varepsilon^{2},
\end{aligned}
$$

so that $\boldsymbol{a} \in \Lambda_{2}^{\varphi}(f)$ and $\boldsymbol{a}^{(m)} \rightarrow \boldsymbol{a}$.

Since $\mathbb{Q}_{0}^{\infty}$ is dense, $\left(\Lambda_{2}^{\varphi}(f), \delta_{f}\right)$ is separable.

Lemma 21. For $f(\neq 0) \in L_{2}$, there exists $G>0$ such that

$$
\delta_{f}(\boldsymbol{a}, \boldsymbol{b}) \leq G d_{2}^{f}(\boldsymbol{a}, \boldsymbol{b}), \quad \boldsymbol{a}, \boldsymbol{b} \in \mathbb{R}^{\infty} .
$$

Proof. Since $\Lambda_{2}(f) \subset \Lambda_{2}^{\varphi}(f)$, by [3, Lemma 4.3]

$$
d_{2}^{f}(\boldsymbol{a}, \mathbf{0})^{2}=\sum_{k} \int_{-\infty}^{+\infty}\left|f\left(x-a_{k}\right)-f(x)\right|^{2} d x<+\infty, \quad a_{k} \in \mathbb{R}
$$

implies

$$
\delta_{f}(\boldsymbol{a}, \mathbf{0})^{2}=\sum_{k} \zeta_{f}\left(a_{k}\right)<+\infty .
$$

Since $\zeta_{f}(u)$ is bounded by Lemma 15 , applying Lemma 4 to $\Phi(u)=\int_{-\infty}^{+\infty} \mid f(x-u)-$ $\left.f(x)\right|^{2} d x, \Psi(u)=\zeta_{f}(u), u \in \mathfrak{X}:=\mathbb{R}$, we have for a constant $C>0$,

$$
\zeta_{f}(u) \leq C \int_{-\infty}^{+\infty}|f(x-u)-f(x)|^{2} d x, \quad u \in \mathbb{R}
$$

which implies

$$
\delta_{f}(\boldsymbol{a}, \mathbf{0}) \leq \sqrt{C} d_{2}^{f}(\boldsymbol{a}, \mathbf{0}), \quad \boldsymbol{a} \in \mathbb{R}^{\infty} .
$$

Corollary 22. For $f(\neq 0) \in L_{2}$, the inclusion $\Lambda_{2}(f) \subset \Lambda_{2}^{\varphi}(f)$ is continuous.

THEOREM 23. For $f(\neq 0) \in L_{2}$, the following two conditions are equivalent:

(i) $\Lambda_{2}(f)=\Lambda_{2}^{\varphi}(f)$;

(ii) $\delta_{f}$ is equivalent to $d_{2}^{f}$ on $\Lambda_{2}(f)$.

Proof. We show (i) $\Rightarrow$ (ii).

Assume $\Lambda_{2}^{\varphi}(f)=\Lambda_{2}(f)$. Then we have $\Lambda_{2}^{\varphi}(f) \subset \Lambda_{2}(f)$, that is,

$$
\delta_{f}(\boldsymbol{a}, \mathbf{0})^{2}=\sum_{k} \zeta_{f}\left(a_{k}\right)<+\infty, \quad a_{k} \in \mathbb{R}
$$

implies

$$
d_{2}^{f}(\boldsymbol{a}, \mathbf{0})^{2}=\sum_{k} \int_{-\infty}^{+\infty}\left|f\left(x-a_{k}\right)-f(x)\right|^{2} d x<+\infty .
$$

Applying Lemma 4 to $\Phi(u)=\zeta_{f}(u), \Psi(u)=\int_{-\infty}^{+\infty}|f(x-u)-f(x)|^{2} d x, u \in \mathfrak{X}:=\mathbb{R}$, we have for a constant $C>0$,

$$
\int_{-\infty}^{+\infty}|f(x-u)-f(x)|^{2} d x \leq C \zeta_{f}(u), \quad u \in \mathbb{R},
$$


which implies $d_{2}^{f}(\boldsymbol{a}, \boldsymbol{b}) \leq \sqrt{C} \delta_{f}(\boldsymbol{a}, \boldsymbol{b})$. On the other hand, by Lemma 21 we have $\delta_{f}(\boldsymbol{a}, \boldsymbol{b}) \leq G d_{2}^{f}(\boldsymbol{a}, \boldsymbol{b})$, which implies (ii).

Conversely assume (ii). Then there exists $B \geq 1$ such that

$$
d_{2}^{f}(\boldsymbol{a}, \mathbf{0}) \leq B \delta_{f}(\boldsymbol{a}, \mathbf{0}), \quad \boldsymbol{a} \in \Lambda_{2}(f) .
$$

For any $\boldsymbol{b}=\left\{b_{k}\right\} \in \Lambda_{2}^{\varphi}(f)$, set $\boldsymbol{b}_{n}=\left\{b_{1}, b_{2}, \ldots, b_{n}, 0,0, \ldots\right\}$. Then $\boldsymbol{b}_{n} \in \Lambda_{2}(f)$ and

$$
d_{2}^{f}(\boldsymbol{b}, \mathbf{0})=\lim _{n \rightarrow+\infty} d_{2}^{f}\left(\boldsymbol{b}_{n}, \mathbf{0}\right) \leq B \lim _{n \rightarrow+\infty} \delta_{f}\left(\boldsymbol{b}_{n}, \mathbf{0}\right)=B \delta_{f}(\boldsymbol{b}, \mathbf{0})<+\infty,
$$

which implies $\boldsymbol{b} \in \Lambda_{2}(f)$ and $\Lambda_{2}^{\varphi}(f) \subset \Lambda_{2}(f)$. The inclusion $\Lambda_{2}(f) \subset \Lambda_{2}^{\varphi}(f)$ is proved in [3, Lemma 4.3], so that we have $\Lambda_{2}(f)=\Lambda_{2}^{\varphi}(f)$.

In the above case, $\left(\Lambda_{2}^{\varphi}(f), \delta_{f}\right)$ is a visible sequential representation of $\left(\Lambda_{2}(f), d_{2}^{f}\right)$.

\section{4. $\quad \Lambda_{2}^{0}(f)=\Lambda_{2}^{\varphi}(f)$ implies $H\left(\varphi_{f}\right)<2$}

THEOREM 24. For $f(\neq 0) \in L_{2}$, the following are equivalent.

(C.1) We have $\Lambda_{2}^{0}(f)=\Lambda_{2}^{\varphi}(f)$, so that $\Lambda_{2}(f)$ is a linear space.

(C.2) There exist $K, L>0$ such that

$$
\int_{X}^{+\infty}|\hat{f}(\alpha)|^{2} d \alpha \leq K \frac{\varphi_{f}(X)}{X^{2}}, \quad X>L .
$$

(C.3) We have $H\left(\varphi_{f}\right)<2$, that is, there exists $\varepsilon>0$ such that

$$
D\left(2-\varepsilon ; \varphi_{f}\right)=\limsup _{X \rightarrow+\infty} \sup _{T \geq 1} \frac{\varphi_{f}(T X)}{\varphi_{f}(X) T^{2-\varepsilon}}<+\infty .
$$

(C.4) There exists $r>0$ such that

$$
\limsup _{X \rightarrow+\infty} \sup _{T \geq 1} \frac{\varphi_{f}(T X)(\log T)^{r}}{\varphi_{f}(X) T^{2}}<+\infty .
$$

(C.5) There exists a non-negative function $\kappa(x), x>0$ such that

$$
\int_{0}^{+\infty} \frac{d x}{\kappa(x)}<+\infty
$$

and

$$
\limsup _{X \rightarrow+\infty} \sup _{T \geq 1} \frac{\varphi_{f}(T X) \kappa(\log T)}{\varphi_{f}(X) T^{2}}<+\infty .
$$

Before proving Theorem 24 we prepare the following lemma.

Lemma 25. Assume (C.2). Then we have

$$
\frac{\varphi_{f}(X)}{X^{2}} \geq \frac{1}{n !}\left(\frac{2}{K+1}\right)^{n+1} \int_{X}^{+\infty} \frac{\varphi_{f}(u)}{u^{3}}\left(\log \frac{u}{X}\right)^{n} d u, \quad X>L
$$

for $n=0,1,2, \ldots$ 
Proof. For any $T \geq 1, X>L$, we have

$$
\begin{aligned}
K \frac{\varphi_{f}(X)}{X^{2}} & \geq \int_{X}^{+\infty}|\hat{f}(\alpha)|^{2} d \alpha=\int_{X}^{+\infty} \frac{\varphi_{f}^{\prime}(\alpha)}{\alpha^{2}} d \alpha \\
& =-\frac{\varphi_{f}(X)}{X^{2}}+2 \int_{X}^{+\infty} \frac{\varphi_{f}(\alpha)}{\alpha^{3}} d \alpha,
\end{aligned}
$$

where $\varphi_{f}(\infty) / \infty^{2}=0$ (see [3, Lemma 4.4]). Therefore, we have

$$
\frac{\varphi_{f}(X)}{X^{2}} \geq \frac{2}{K+1} \int_{X}^{+\infty} \frac{\varphi_{f}(\alpha)}{\alpha^{3}} d \alpha, \quad X>L,
$$

which shows $\left(\Theta_{0}\right)$.

Assume $\left(\Theta_{n}\right)$. Then by $\left(\Theta_{0}\right)$, we have

$$
\frac{\varphi_{f}(u)}{u^{3}} \geq \frac{2}{K+1} \frac{1}{u} \int_{u}^{+\infty} \frac{\varphi_{f}(s)}{s^{3}} d s, \quad u>L,
$$

and changing the order of integration, we have for $X>L$,

$$
\begin{aligned}
\frac{\varphi_{f}(X)}{X^{2}} & \geq \frac{1}{n !}\left(\frac{2}{K+1}\right)^{n+1} \int_{X}^{+\infty} \frac{\varphi_{f}(u)}{u^{3}}\left(\log \frac{u}{X}\right)^{n} d u \\
& \geq \frac{1}{n !}\left(\frac{2}{K+1}\right)^{n+2} \int_{X}^{+\infty}\left(\log \frac{u}{X}\right)^{n} \frac{d u}{u} \int_{u}^{+\infty} \frac{\varphi_{f}(s)}{s^{3}} d s \\
& =\frac{1}{(n+1) !}\left(\frac{2}{K+1}\right)^{n+2} \int_{X}^{+\infty} \frac{\varphi_{f}(s)}{s^{3}}\left(\log \frac{s}{X}\right)^{n+1} d s
\end{aligned}
$$

which shows $\left(\Theta_{n+1}\right)$.

Thus, the lemma is proved by mathematical induction.

Proof of Theorem 24. (C.1) $\Rightarrow$ (C.2) Assume $\Lambda_{2}^{0}(f)=\Lambda_{2}^{\varphi}(f)$. Since $\hat{f} \neq 0$ there exists $L>0$ such that $\varphi_{f}(L)>0$. By $\Lambda_{2}^{0}(f)=\Lambda_{2}^{\varphi}(f)$ and Lemma 1 ,

$$
\sum_{k} a_{k}^{2}\left[1+\varphi_{f}\left(\frac{1}{\left|a_{k}\right|}\right)\right]<+\infty, \quad a_{k} \in \mathbb{R}
$$

implies

$$
\sum_{k} \int_{1 /\left|a_{k}\right|}^{+\infty}|\hat{f}(\alpha)|^{2} d \alpha<+\infty
$$

Since $\int_{1 /|u|}^{+\infty}|\hat{f}(\alpha)|^{2} d \alpha$ is bounded, Lemma 4 is applicable to $\Phi(u)=u^{2}\left(1+\varphi_{f}(1 /|u|)\right)$ and $\Psi(u)=\int_{1 /|u|}^{+\infty}|\hat{f}(\alpha)|^{2} d \alpha, u \in \mathfrak{X}:=\mathbb{R}$. Hence, there exists $C>0$ such that for any $X>L$,

$$
\int_{X}^{+\infty}|\hat{f}(\alpha)|^{2} d \alpha \leq C \frac{1}{X^{2}}\left(1+\varphi_{f}(X)\right) \leq C\left(\frac{1}{\varphi_{f}(L)}+1\right) \frac{\varphi_{f}(X)}{X^{2}}
$$

which implies (C.2) with $K:=C\left(1 / \varphi_{f}(L)+1\right)$.

(C.2) $\Rightarrow$ (C.1) This is derived from Lemma 1 . 
(C.2) $\Rightarrow$ (C.3) Assume (C.2). Since $\varphi_{f}(X)$ is a non-decreasing function, by Lemma 25 , for $n=0,1,2, \ldots$, and for $T \geq 1$, we have

$$
\begin{aligned}
\frac{\varphi_{f}(X)}{X^{2}} & \geq \frac{1}{n !}\left(\frac{2}{K+1}\right)^{n+1} \int_{X}^{+\infty} \frac{\varphi_{f}(u)}{u^{3}}\left(\log \frac{u}{X}\right)^{n} d u \\
& \geq \frac{1}{n !}\left(\frac{2}{K+1}\right)^{n+1} \int_{T X}^{+\infty} \frac{\varphi_{f}(u)}{u^{3}}\left(\log \frac{u}{X}\right)^{n} d u \\
& \geq \frac{1}{n !}\left(\frac{2}{K+1}\right)^{n+1} \varphi_{f}(T X)(\log T)^{n} \int_{T X}^{+\infty} \frac{d u}{u^{3}} \\
& =\frac{1}{n !}\left(\frac{2}{K+1}\right)^{n+1} \frac{\varphi_{f}(T X)(\log T)^{n}}{2 T^{2} X^{2}},
\end{aligned}
$$

which implies

$$
(K+1) \frac{1}{2^{n}} \geq \frac{\varphi_{f}(T X)}{\varphi_{f}(X) T^{2}} \frac{1}{n !}\left(\frac{\log T}{K+1}\right)^{n}, \quad X>L, T \geq 1 .
$$

Summing up on both sides with respect to $n=0,1,2, \ldots$, we have

$$
2(K+1) \geq \frac{\varphi_{f}(T X)}{\varphi_{f}(X) T^{2}} e^{\log T /(K+1)}=\frac{\varphi_{f}(T X)}{\varphi_{f}(X) T^{2-1 /(K+1)}}, \quad X>L, T \geq 1,
$$

so that

$$
D\left(2-\frac{1}{K+1} ; \varphi_{f}\right) \leq 2(K+1)<+\infty .
$$

(C.3) $\Rightarrow$ (C.4) This is trivial.

(C.4) $\Rightarrow$ (C.5) Assume (C.4). Then there exist $K, L>0$ such that

$$
\frac{\varphi_{f}(T X)(\log T)^{r}}{\varphi_{f}(X) T^{2}} \leq K, \quad X \geq L, T \geq 1 .
$$

Since $\varphi_{f}(x)$ is non-decreasing, for $1 \leq T \leq e$ we have

$$
\begin{aligned}
\frac{\varphi_{f}(T X)}{\varphi_{f}(X) T^{2}} & \leq \frac{\varphi_{f}(e X)}{\varphi_{f}(X)}=e^{2} \frac{\varphi_{f}(e X)(\log e)^{r}}{\varphi_{f}(X) e^{2}} \\
& \leq e^{2} \sup _{T \geq 1} \frac{\varphi_{f}(T X)(\log T)^{r}}{\varphi_{f}(X) T^{2}} \leq e^{2} K, \quad X \geq L .
\end{aligned}
$$

On the other hand, for $n_{0}:=\langle\langle 1 / r\rangle+1$ we have

$$
\begin{aligned}
\varphi_{f}(T X) & =\varphi_{f}\left(T^{1 / n_{0}}\left(T^{1-1 / n_{0}} X\right)\right) \leq K \frac{T^{2 / n_{0}} \varphi_{f}\left(T^{1-1 / n_{0}} X\right)}{\left(\log T^{1 / n_{0}}\right)^{r}} \\
& =K \frac{T^{2 / n_{0}} \varphi_{f}\left(T^{1 / n_{0}}\left(T^{1-2 / n_{0}} X\right)\right)}{\left(\log T^{1 / n_{0}}\right)^{r}} \leq K^{2} \frac{T^{4 / n_{0}} \varphi_{f}\left(T^{1-2 / n_{0}} X\right)}{\left(\log T^{1 / n_{0}}\right)^{2 r}} \\
& \leq \cdots \leq K^{n_{0}} \frac{T^{2 n_{0} / n_{0}} \varphi_{f}(X)}{\left(\log T^{1 / n_{0}}\right)^{n_{0} r}}=K^{n_{0}} n_{0}^{n_{0} r} \frac{T^{2} \varphi_{f}(X)}{(\log T)^{n_{0} r}} .
\end{aligned}
$$


Consequently, we have

$$
\limsup _{X \rightarrow+\infty} \sup _{T \geq 1} \frac{\varphi_{f}(T X)\left[1+(\log T)^{n_{0} r}\right]}{\varphi_{f}(X) T^{2}}<+\infty,
$$

which shows (C.5) with $\kappa(x):=1+x^{n_{0} r}$.

(C.5) $\Rightarrow$ (C.2) Assume (C.5). Then there exist $K, L>0$ such that

$$
\varphi_{f}(T X) \kappa(\log T) \leq K \varphi_{f}(X) T^{2}, \quad X \geq L .
$$

Utilizing integration by parts, for $X>L$ we have

$$
\begin{aligned}
\int_{X}^{+\infty}|\hat{f}(\alpha)|^{2} d \alpha & =\int_{X}^{+\infty} \frac{\varphi_{f}^{\prime}(\alpha)}{\alpha^{2}} d \alpha=-\frac{\varphi_{f}(X)}{X^{2}}+2 \int_{X}^{+\infty} \frac{\varphi_{f}((\alpha / X) X)}{\alpha^{3}} d \alpha \\
& \leq-\frac{\varphi_{f}(X)}{X^{2}}+2 K \int_{X}^{+\infty}\left(\frac{\alpha}{X}\right)^{2} \frac{\varphi_{f}(X)}{\alpha^{3} \kappa(\log (\alpha / X))} d \alpha \\
& =\left(2 K \int_{0}^{+\infty} \frac{1}{\kappa(u)} d u-1\right) \frac{\varphi_{f}(X)}{X^{2}} .
\end{aligned}
$$

We remark

$$
2 K \int_{0}^{+\infty} \frac{1}{\kappa(u)} d u-1 \geq 0
$$

since

$$
\begin{aligned}
\int_{0}^{+\infty} \frac{1}{\kappa(u)} d u & =\int_{0}^{+\infty} \frac{d u}{\kappa\left(\log e^{u}\right)} \geq \int_{0}^{+\infty} \frac{\varphi_{f}\left(e^{u} X\right)}{K \varphi_{f}(X) e^{2 u}} d u \\
& =\frac{X^{2}}{K \varphi_{f}(X)} \int_{X}^{+\infty} \frac{\varphi_{f}(\alpha)}{\alpha^{3}} d \alpha \geq \frac{X^{2} \varphi_{f}(X)}{K \varphi_{f}(X)} \int_{X}^{+\infty} \frac{d \alpha}{\alpha^{3}}=\frac{1}{2 K}
\end{aligned}
$$

\section{Example}

In the previous section, we proved that $H\left(\varphi_{f}\right)<2$ is a necessary and sufficient condition for $\Lambda_{2}^{0}(f)=\Lambda_{2}^{\varphi}(f)$ which implies the linearity of $\Lambda_{2}(f)$. But $\Lambda_{2}^{0}(f)=\Lambda_{2}^{\varphi}(f)$ is not a necessary condition for the linearity of $\Lambda_{2}(f)$. In fact, we have the following illuminating example of $\theta \in L_{2}$ such that both $\Lambda_{2}(\theta)$ and $\Lambda_{2}^{\varphi}(\theta)$ are linear but $\Lambda_{2}^{0}(\theta) \neq \Lambda_{2}^{\varphi}(\theta)$.

EXAMPLE 26. Define $\theta \in L_{2}$ by

$$
\hat{\theta}(\alpha):= \begin{cases}\frac{1}{\sqrt{|\alpha|} \log |\alpha|}, & |\alpha| \geq e, \\ 0, & |\alpha|<e,\end{cases}
$$

a function $l(x)$ by

$$
l(x):= \begin{cases}\frac{1}{\log ((1 /|x|) \vee e)}, & x \neq 0, \\ 0, & x=0,\end{cases}
$$

and

$$
\rho_{1}^{\theta}(\boldsymbol{a}, \boldsymbol{b}):=\sqrt{\sum_{k} l\left(a_{k}-b_{k}\right)}, \quad \rho_{2}^{\theta}(\boldsymbol{a}, \boldsymbol{b}):=\sqrt{\sum_{k} l\left(a_{k}-b_{k}\right)^{2}}, \quad \boldsymbol{a}, \boldsymbol{b} \in \mathbb{R}^{\infty},
$$


where $x \vee y:=\max \{x, y\}$. Then we have

$$
\Lambda_{2}^{0}(\theta)=\left\{\boldsymbol{a}=\left\{a_{k}\right\} \in \mathbb{R}^{\infty} \mid \sum_{k} l\left(a_{k}\right)<+\infty\right\},
$$

which is a linear space, and have

$$
\Lambda_{2}^{\varphi}(\theta)=\left\{\boldsymbol{a}=\left\{a_{k}\right\} \in \mathbb{R}^{\infty} \mid \sum_{k} l\left(a_{k}\right)^{2}<+\infty\right\},
$$

which is also a linear space, but $\Lambda_{2}^{0}(\theta) \neq \Lambda_{2}^{\varphi}(\theta)$.

Furthermore, $\rho_{1}^{\theta}$ and $\rho_{2}^{\theta}$ are metrics. The quasi-metric $\gamma_{\theta}$, the metrics $d_{2}^{f}$ and $\rho_{1}^{\theta}$ are mutually equivalent on $\Lambda_{2}^{0}(\theta)$.

On the other hand, the quasi-metric $\delta_{\theta}$ and the metric $\rho_{2}^{\theta}$ are equivalent on $\Lambda_{2}^{\varphi}(\theta)$.

Proof. Since $|\hat{\theta}(\alpha)|$ is non-increasing for $\alpha \geq e, \Lambda_{2}(\theta)$ is a linear space [3, Corollary 4.2] and $\Lambda_{2}(\theta)=\Lambda_{2}^{0}(\theta)$ holds.

First, we estimate $\gamma_{\theta}(\boldsymbol{a}, \mathbf{0})$. We have for $\boldsymbol{a} \in \Lambda_{2}^{0}(\theta)$,

$$
\begin{aligned}
2 \gamma_{\theta}(\boldsymbol{a}, \mathbf{0})^{2} & =\sum_{k} a_{k}^{2} \varphi_{\theta}\left(\frac{1}{\left|a_{k}\right|}\right)+\sum_{k} \int_{1 /\left|a_{k}\right|}^{+\infty}|\hat{\theta}(\alpha)|^{2} d \alpha \geq \sum_{k} \int_{1 /\left|a_{k}\right|}^{+\infty}|\hat{\theta}(\alpha)|^{2} d \alpha \\
& =\sum_{k} \int_{\left(1 /\left|a_{k}\right|\right) \vee e}^{+\infty}\left(\frac{1}{\sqrt{\alpha} \log \alpha}\right)^{2} d \alpha=\sum_{k} l\left(a_{k}\right)=\rho_{1}^{\theta}(\boldsymbol{a}, \mathbf{0})^{2} .
\end{aligned}
$$

On the other hand, remarking

$$
\frac{e^{2}}{4} \leq \frac{\alpha}{(\log \alpha)^{2}} \leq \frac{4}{e} \frac{X}{(\log X)^{2}}, \quad e \leq \alpha \leq X,
$$

we have

$$
\begin{aligned}
& \sum_{k} a_{k}^{2} \varphi_{\theta}\left(\frac{1}{\left|a_{k}\right|}\right) \\
& \quad=\sum_{k: 1 /\left|a_{k}\right|>e} a_{k}^{2} \int_{e}^{1 /\left|a_{k}\right|} \frac{\alpha}{(\log \alpha)^{2}} d \alpha \\
& \quad \leq \sum_{k: 1 /\left|a_{k}\right|>e} \frac{4}{e} a_{k}^{2} \frac{1}{\left|a_{k}\right|\left(\log \left(1 /\left|a_{k}\right|\right)\right)^{2}}\left(\frac{1}{\left|a_{k}\right|}-e\right) \leq 3 \sum_{k: 1 /\left|a_{k}\right|>e} \frac{1}{\left(\log \left(1 /\left|a_{k}\right|\right)\right)^{2}} \\
& \quad \leq 3 \sum_{k} l\left(a_{k}\right),
\end{aligned}
$$

so that

$$
\begin{aligned}
2 \gamma_{\theta}(\boldsymbol{a}, \mathbf{0})^{2} & =\sum_{k} a_{k}^{2} \varphi_{\theta}\left(\frac{1}{\left|a_{k}\right|}\right)+\sum_{k} \int_{1 /\left|a_{k}\right|}^{+\infty}|\hat{\theta}(\alpha)|^{2} d \alpha \leq 4 \sum_{k} l\left(a_{k}\right) \\
& =4 \rho_{1}^{\theta}(\boldsymbol{a}, \mathbf{0})^{2} .
\end{aligned}
$$

Consequently, we have

$$
\rho_{1}^{\theta}(\boldsymbol{a}, \boldsymbol{b}) \leq \sqrt{2} \gamma_{\theta}(\boldsymbol{a}, \boldsymbol{b}) \leq 2 \rho_{1}^{\theta}(\boldsymbol{a}, \boldsymbol{b}), \quad \boldsymbol{a}, \boldsymbol{b} \in \Lambda_{2}^{0}(\theta)
$$


and

$$
\Lambda_{2}(\theta)=\Lambda_{2}^{0}(\theta)=\left\{\boldsymbol{a}=\left\{a_{k}\right\} \in \mathbb{R}^{\infty} \mid \sum_{k} l\left(a_{k}\right)<+\infty\right\} .
$$

By Theorem 10, $\gamma_{\theta}$ and $d_{2}^{\theta}$ are equivalent on $\Lambda_{2}^{0}(\theta)$.

Next, we show $D\left(2 ; \varphi_{\theta}\right)<+\infty$. In fact, for $x \geq 2 e$ and $T \geq 1$ we have

$$
\begin{aligned}
\varphi_{\theta}(T x) & =\int_{e}^{T x} \frac{\alpha}{(\log \alpha)^{2}} d \alpha=\int_{e / T}^{e} \frac{T^{2} u}{(\log T u)^{2}} d u+\int_{e}^{x} \frac{T^{2} u}{(\log T u)^{2}} d u \\
& \leq T^{2} e\left(e-\frac{e}{T}\right)+\int_{e}^{x} \frac{T^{2} u}{(\log u)^{2}} d u \leq T^{2} e^{2}+T^{2} \varphi_{\theta}(x),
\end{aligned}
$$

which implies

$$
\frac{\varphi_{\theta}(T x)}{\varphi(x) T^{2}} \leq \frac{e^{2}}{\varphi_{\theta}(x)}+1 \leq \frac{e^{2}}{\varphi_{\theta}(2 e)}+1<+\infty,
$$

that is, $D\left(2 ; \varphi_{\theta}\right)<+\infty$. Hence, by Theorem $20, \Lambda_{2}^{\varphi}(\theta)$ is a topological linear space with the linear quasi-metric $\delta_{\theta}$.

Furthermore, we estimate $\delta_{\theta}(\boldsymbol{a}, \mathbf{0})$. We have

$$
\sum_{k}\left(\left|a_{k}\right| \wedge 1\right)^{2} \leq \sum_{k:\left|a_{k}\right|<1 / e} \frac{1}{\left[\log \left(\left(1 /\left|a_{k}\right|\right) \vee e\right)\right]^{2}}+\sum_{k:\left|a_{k}\right| \geq 1 / e} 1=\sum_{k} l\left(a_{k}\right)^{2}
$$

and

$$
\sum_{k}\left(\left|a_{k}\right| \wedge 1\right)^{2} \varphi_{\theta}\left(\frac{1}{\left|a_{k}\right| \wedge 1}\right)=\sum_{k: 1 /\left|a_{k}\right|>e} a_{k}^{2} \varphi_{\theta}\left(\frac{1}{\left|a_{k}\right|}\right) \leq 3 \sum_{k: 1 /\left|a_{k}\right|>e} l\left(a_{k}\right)^{2} .
$$

Therefore, we have

$$
\delta_{\theta}(\boldsymbol{a}, \mathbf{0})^{2}=\sum_{k}\left(\left|a_{k}\right| \wedge 1\right)^{2}\left[1+\varphi_{\theta}\left(\frac{1}{\left|a_{k}\right| \wedge 1}\right)\right] \leq 4 \sum_{k} l\left(a_{k}\right)^{2}=4 \rho_{2}^{\theta}(\boldsymbol{a}, \mathbf{0})^{2} .
$$

Concerning the lower estimation of $\delta_{\theta}(\boldsymbol{a}, \mathbf{0})$, since $\alpha /(\log \alpha)^{2}$ is non-decreasing for $x \geq e^{2}$, we have

$$
\begin{aligned}
\delta_{\theta}(\boldsymbol{a}, \mathbf{0})^{2} & =\sum_{k}\left(\left|a_{k}\right| \wedge 1\right)^{2}\left[1+\varphi_{\theta}\left(\frac{1}{\left|a_{k}\right| \wedge 1}\right)\right] \\
& \geq \sum_{k: 1 /\left(2 e^{2}\right)<\left|a_{k}\right|}\left(\left|a_{k}\right| \wedge 1\right)^{2}+\sum_{k:\left|a_{k}\right|<1 /\left(2 e^{2}\right)} a_{k}^{2} \varphi_{\theta}\left(\frac{1}{\left|a_{k}\right|}\right) \\
& \geq \frac{1}{4 e^{4}} \sum_{k: 1 /\left(2 e^{2}\right)<\left|a_{k}\right|} 1+\sum_{k: 1 /\left|a_{k}\right|>2 e^{2}} a_{k}^{2} \int_{1 /\left(2\left|a_{k}\right|\right)}^{1 /\left|a_{k}\right|} \frac{\alpha}{(\log \alpha)^{2}} d \alpha \\
& \geq \frac{1}{4 e^{4}} \sum_{k: 1 /\left(2 e^{2}\right)<\left|a_{k}\right|} 1+\sum_{k: 1 /\left|a_{k}\right| \geq 2 e^{2}} a_{k}^{2} \frac{1}{2\left|a_{k}\right|\left[\log \left(1 /\left(2\left|a_{k}\right|\right)\right)\right]^{2}}\left(\frac{1}{\left|a_{k}\right|}-\frac{1}{2\left|a_{k}\right|}\right) \\
& \geq \frac{1}{4 e^{4}} \sum_{k: 1 /\left|a_{k}\right|<2 e^{2}} \frac{1}{\left[\log \left(\left(1 /\left|a_{k}\right|\right) \vee e\right)\right]^{2}}+\frac{1}{4} \sum_{k: 1 /\left|a_{k}\right| \geq 2 e^{2}} \frac{1}{\left(\log \left(1 /\left|a_{k}\right|\right)\right)^{2}} \\
& \geq \frac{1}{18^{2}} \sum_{k} l\left(a_{k}\right)^{2}=\frac{1}{18^{2}} \rho_{2}^{\theta}(\boldsymbol{a}, \mathbf{0})^{2} .
\end{aligned}
$$


Consequently, we have

$$
\frac{1}{18} \rho_{2}^{\theta}(\boldsymbol{a}, \boldsymbol{b}) \leq \delta_{\theta}(\boldsymbol{a}, \boldsymbol{b}) \leq 2 \rho_{2}^{\theta}(\boldsymbol{a}, \boldsymbol{b}), \quad \boldsymbol{a}, \boldsymbol{b} \in \Lambda_{2}^{\varphi}(\theta)
$$

and

$$
\Lambda_{2}^{\varphi}(\theta)=\left\{\boldsymbol{a}=\left\{a_{k}\right\} \in \mathbb{R}^{\infty} \mid \sum_{k} l\left(a_{k}\right)^{2}<+\infty\right\} .
$$

Finally, we show that $\rho_{1}^{\theta}$ and $\rho_{2}^{\theta}$ are metrics. By elementary estimations we have

$$
l(x+y) \leq l(x)+l(y)
$$

for any $x, y \in \mathbb{R}$. Therefore, we have for any $\boldsymbol{a}, \boldsymbol{b} \in \mathbb{R}^{\infty}$,

$$
\begin{aligned}
\rho_{1}^{\theta}(\boldsymbol{a}+\boldsymbol{b}, \mathbf{0}) & =\sqrt{\sum_{k} l\left(a_{k}+b_{k}\right)} \leq \sqrt{\sum_{k} l\left(a_{k}\right)+\sum_{k} l\left(b_{k}\right)} \\
& \leq \sqrt{\sum_{k} l\left(a_{k}\right)}+\sqrt{\sum_{k} l\left(b_{k}\right)}=\rho_{1}^{\theta}(\boldsymbol{a}, \mathbf{0})+\rho_{1}^{\theta}(\boldsymbol{b}, \mathbf{0})
\end{aligned}
$$

and

$$
\begin{aligned}
\rho_{2}^{\theta}(\boldsymbol{a}+\boldsymbol{b}, \mathbf{0}) & =\sqrt{\sum_{k} l\left(a_{k}+b_{k}\right)^{2}} \leq \sqrt{\sum_{k}\left[l\left(a_{k}\right)+l\left(b_{k}\right)\right]^{2}} \\
& \leq \rho_{2}^{\theta}(\boldsymbol{a}, \mathbf{0})+\rho_{2}^{\theta}(\boldsymbol{b}, \mathbf{0}) .
\end{aligned}
$$

\section{REFERENCES}

[1] S. D. Chatterji and V. Mandrekar. Quasi-invariance of measures under translation. Math. Z. 154 (1977), 19-29.

[2] A. Honda, Y. Okazaki and H. Sato. An $L_{p}$ function determines $\ell_{p}$. Proc. Japan Acad. Ser. A 84(3) (2008), 39-41.

[3] A. Honda, Y. Okazaki and H. Sato. Doubling condition and linearity of the sequence space $\Lambda_{p}(f)$. Kyushu J. Math. 65(2) (2011), 335-347.

[4] A. Honda, Y. Okazaki and H. Sato. Metrics on the sequence space $\Lambda_{p}(f)$. Kyushu J. Math. 66(2) (2012), 365-374.

[5] A. Hora. Quasi-invariant measures on commutative Lie groups of infinite product type. Math. Z. 206(1) (1991), 169-192.

[6] G. Nakamura and K. Hashimoto. On the linearity of some sets of sequences defined by $L_{p}$-functions and $L_{1}$-functions determining $\ell_{1}$. Proc. Japan Acad. Ser. A 87(5) (2011), 77-82.

[7] L. A. Shepp. Distinguishing a sequence of random variables from a translate of itself. Ann. Math. Statist. 36(4) (1965), 1107-1112.

[8] H. Shimomura. An aspect of quasi-invariant measure on $\mathbb{R}^{\infty}$. Publ. Res. Inst. Math. Sci. 11 (1976), 749-773.

[9] Y. Yamasaki. Measures on infinite dimensional spaces. World Scientific, Singapore, 1985. 
Aoi Honda

Department of Systems Design and Informatics

Kyushu Institute of Technology

680-4, Kawazu, Iizuka 820-8502, Japan

(E-mail:aoi@ces.kyutech.ac.jp)

Yoshiaki Okazaki

Fuzzy Logic Systems Institute (FLSI)

680-41, Kawazu, Iizuka 820-0067, Japan

(E-mail: okazaki@flsi.or.jp)

\section{Hiroshi Sato}

Faculty of Mathematics

Kyushu University

744 Motooka, Nishiku Fukuoka, 819-0395, Japan

(E-mail: satohrs@etude.ocn.ne.jp) 